TRANSACTIONS OF THE

AMERICAN MATHEMATICAL SOCIETY

Volume 358, Number 2, Pages 871-891

S 0002-9947(05)03800-6

Article electronically published on April 22, 2005

\title{
NONDEGENERATE $q$-BIRESOLVING TEXTILE SYSTEMS AND EXPANSIVE AUTOMORPHISMS OF ONESIDED FULL SHIFTS
}

\author{
MASAKAZU NASU
}

\begin{abstract}
We study nondegenerate, $q$-biresolving textile systems and using properties of them, we prove a conjecture of Boyle and Maass on arithmetic constraints for expansive automorphisms of onesided full shifts and positively expansive endomorphisms of mixing topological Markov shifts. A similar result is also obtained for expansive leftmost-permutive endomorphisms of onesided full shifts.
\end{abstract}

\section{INTRODUCTION}

In [BM, Conjectures 7.1, 7.2 and 7.3], Boyle and Maass gave three conjectures on expansive automorphisms of onesided full shifts. The first conjecture was the one that the dynamical system defined by an expansive automorphism of a onesided full shift is topologically conjugate to a topological Markov shift. It was proved in [N4. Therefore, if $\tilde{\varphi}$ is an expansive automorphism of the onesided full $N$ shift $\left(A^{\mathbf{N}}, \tilde{\sigma}_{A}\right)$ over the alphabet $A$ of $N$ symbols, then there exists an $N$-to-one, positively expansive endomorphism $\varphi_{0}$ of a (twosided) topological Markov shift $\left(X_{0}, \sigma_{0}\right)$ such that $\left(A^{\mathbf{N}}, \tilde{\varphi}\right)$ and $\left(X_{0}, \sigma_{0}\right)$ are topologically conjugate and so are $\left(A^{\mathbf{N}}, \tilde{\sigma}_{A}\right)$ and $\left(X_{0}, \varphi_{0}\right)$ through a common homeomorphism of $A^{\mathbf{N}}$ onto $X_{0}$. Boyle and Maass proved that in this situation the topological Markov shift $\left(X_{0}, \sigma_{0}\right)$ is shift equivalent to some full $J$-shift such that (i) $J$ and $N$ are divisible by the same primes, and gave the second conjecture that in the same situation $\left(A^{\mathbf{N}}, \tilde{\varphi}\right)$ is topologically conjugate to a full shift. The third conjecture of Boyle and Maass is stated as follows. In the same situation, the left and right multipliers $l_{\varphi_{0}}$ and $r_{\varphi_{0}}$ (developed by Boyle [B]) are integers such that all $N, l_{\varphi_{0}}$ and $r_{\varphi_{0}}$ are divisible by the same primes, and in particular (ii) if a prime $p$ divides $N$, then $p^{2}$ divides $N$. $\left(l_{\varphi_{0}}\right.$ and $r_{\varphi_{0}}$ are independent of the choice of $\varphi_{0}$ and have the property that $N=l_{\varphi_{0}} r_{\varphi_{0}}$ $([\mathrm{B}])$.) Boyle and Maass $[\mathrm{BM}$ ] proved this conjecture for the case that $N$ is a power of a prime. The result of Boyle and Maass above and the third conjecture imply that (i) and (ii) in them are a necessary condition for positive integers $J$ and $N$ to have the property that there is an automorphism of the onesided full $N$-shift which is conjugate to the full $J$-shift $([\mathrm{BM}]$ ). Boyle and Maass $[\mathrm{BM}]$ showed that (i) and (ii) are a sufficient condition for $J$ and $N$ to have the property.

Received by the editors April 10, 2003 and, in revised form, April 13, 2004.

2000 Mathematics Subject Classification. Primary 37B10; Secondary 37B15, 54H20.

This research was partially supported by Grant-in-Aid for Scientific Research (No. 13440056), Ministry of Education, Culture, Sports, Science and Technology, Japan. 
In this paper, we study nondegenerate, $q$-biresolving textile systems. Using properties of them (Proposition 3.9), we prove the third conjecture of Boyle and Maass and obtain a similar result for expansive leftmost-permutive endomorphisms of onesided full shifts. The second conjecture of Boyle and Maass is still unsettled as far as the author knows.

The results of Boyle and Maass above and their second and third conjectures have alternative equivalent statements (see [N4, Section 6]). The result stated first is equivalent to the statement "A mixing topological Markov shift having a positively expansive $N$-to-one endomorphism is shift equivalent to some full $J$-shift, where $J$ and $N$ are divisible by the same primes". The second conjecture is equivalent to the statement "A mixing topological Markov shift having a positively expansive endomorphism is conjugate to a full shift". The third conjecture is equivalent to the statement "If a positively expansive endomorphism $\varphi$ of a mixing topological Markov shift is $N$-to-one, then $l_{\varphi}$ and $r_{\varphi}$ are integers such that all $N, l_{\varphi}$ and $r_{\varphi}$ are divisible by the same primes, and in particular if a prime $p$ divides $N$, then $p^{2}$ divides $N "$.

The reader is referred to [Ki] or [LM] for a comprehensive introduction to symbolic dynamics, and to $\mathrm{AH}$ for information on topological dynamics.

The author is grateful to the referee for pointing out errors in the manuscript.

This paper was written when the author was with the Graduate School of Engineering, Hiroshima University.

\section{Preliminaries}

Let $A$ be an alphabet (i.e., a nonempty finite set of symbols). Let $A^{\mathbf{Z}}=$ $\left\{\left(a_{j}\right)_{j \in \mathbf{Z}} \mid a_{j} \in A\right\}$ be endowed with a metric compatible with the product topology of the discrete topology on $A$. Let $\sigma_{A}: A^{\mathbf{Z}} \rightarrow A^{\mathbf{Z}}$ be defined by

$$
\sigma_{A}\left(\left(a_{j}\right)_{j \in \mathbf{Z}}\right)=\left(a_{j+1}\right)_{j \in \mathbf{Z}} .
$$

The dynamical system $\left(A^{\mathbf{Z}}, \sigma_{A}\right)$ is called the full shift over $A$. Let $X$ be a closed subset of $A^{\mathbf{Z}}$ with $\sigma_{A}(X)=X$. Let $\sigma=\sigma_{A} \mid X$. Then we have a dynamical system $(X, \sigma)$, which is called a subshift over $A$. Let $A^{\mathbf{N}}=\left\{\left(a_{j}\right)_{j \in \mathbf{N}} \mid a_{j} \in A\right\}$ be endowed with a metric compatible with the product topology of the discrete topology on $A$. Let $\tilde{\sigma}_{A}: A^{\mathbf{N}} \rightarrow A^{\mathbf{N}}$ be defined by

$$
\tilde{\sigma}_{A}\left(\left(a_{j}\right)_{j \in \mathbf{N}}\right)=\left(a_{j+1}\right)_{j \in \mathbf{N}} .
$$

The dynamical system $\left(A^{\mathbf{N}}, \tilde{\sigma}_{A}\right)$ is called the onesided full shift over $A$. For a subshift $(X, \sigma)$ over $A$, let $\tilde{X}=\left\{\left(a_{j}\right)_{j \in \mathbf{N}} \mid \exists\left(a_{j}\right)_{j \in \mathbf{Z}} \in X\right\}$. Then we have a dynamical system $(\tilde{X}, \tilde{\sigma})$ with $\tilde{\sigma}=\tilde{\sigma}_{A} \mid \tilde{X}$, which is called a onesided subshift over $A$ and is said to be induced by $(X, \sigma)$.

Let $G$ be a graph. Here a graph means a directed graph which may have multiple arcs and multiple loops. Let $A_{G}$ and $V_{G}$ denote the arc-set of $G$ and the vertex-set of $G$, respectively. Let $i_{G}: A_{G} \rightarrow V_{G}$ and $t_{G}: A_{G} \rightarrow V_{G}$ be the mappings such that for $a \in A_{G}, i_{G}(a)$ and $t_{G}(a)$ are the initial and terminal vertices, respectively, of $a$ in $G$. Hence the graph $G$ is represented by $V_{G} \stackrel{i_{G}}{\longleftarrow} A_{G} \stackrel{t_{G}}{\longrightarrow} V_{G}$. Let $X_{G}$ be the set of all points $\left(a_{j}\right)_{j \in \mathbf{Z}}$ in $A_{G}^{\mathbf{Z}}$ such that $t_{G}\left(a_{j}\right)=i_{G}\left(a_{j+1}\right)$ for all $j \in \mathbf{Z}$. Then we have a subshift $\left(X_{G}, \sigma_{G}\right)$, which is called the topological Markov shift defined by $G$. 
For graphs $\Gamma$ and $G$, a graph-homomorphism $h$ of $\Gamma$ into $G$, written by $h: \Gamma \rightarrow G$, is a pair $\left(h_{A}, h_{V}\right)$ of mappings $h_{A}: A_{\Gamma} \rightarrow A_{G}$ (arc-map) and $h_{V}: V_{\Gamma} \rightarrow V_{G}$ (vertexmap) such that the following diagram is commutative:

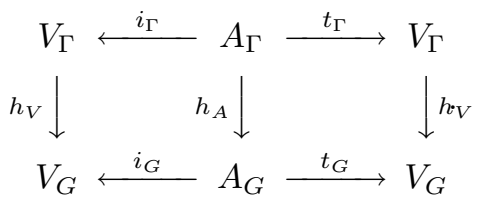

A graph-homomorphism $h: \Gamma \rightarrow G$ gives 1-block maps $\phi_{h}: X_{\Gamma} \rightarrow X_{G}$ and $\tilde{\phi}_{h}: \tilde{X}_{\Gamma} \rightarrow \tilde{X}_{G}$ by

$$
\begin{aligned}
& \phi_{h}\left(\left(\alpha_{j}\right)_{j \in \mathbf{Z}}\right)=\left(h_{A}\left(\alpha_{j}\right)_{j \in \mathbf{Z}}\right), \quad\left(\alpha_{j}\right)_{j \in \mathbf{Z}} \in X_{\Gamma}, \alpha_{j} \in A_{\Gamma}, \\
& \tilde{\phi}_{h}\left(\left(\alpha_{j}\right)_{j \in \mathbf{N}}\right)=\left(h_{A}\left(\alpha_{j}\right)_{j \in \mathbf{N}}\right), \quad\left(\alpha_{j}\right)_{j \in \mathbf{N}} \in \tilde{X}_{\Gamma}, \alpha_{j} \in A_{\Gamma} .
\end{aligned}
$$

Now we recall the notion of a textile system introduced in [N2].

A textile system $T$ (defined) over a graph $G$ is defined to be an ordered pair of graph-homomorphisms $p: \Gamma \rightarrow G$ and $q: \Gamma \rightarrow G$ such that $\alpha \in A_{\Gamma}$ is uniquely determined by the quadruple $\left(i_{\Gamma}(\alpha), t_{\Gamma}(\alpha), p_{A}(\alpha), q_{A}(\alpha)\right)$. We write

$$
T=(p, q: \Gamma \rightarrow G) \text {. }
$$

We have the following commutative diagram:

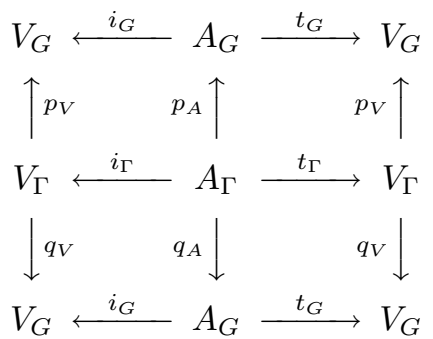

If we observe this diagram vertically, then we have the ordered pair of graphhomomorphisms

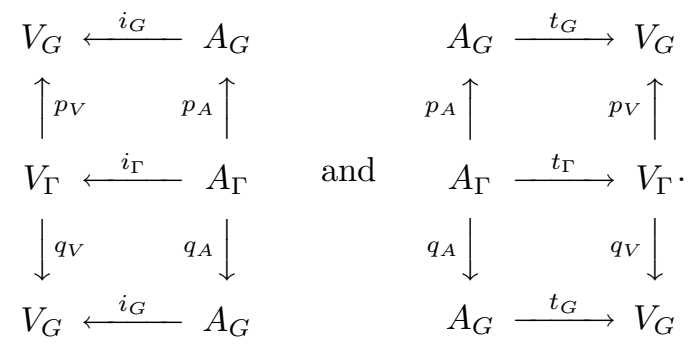

This defines another textile system

$$
T^{*}=\left(p^{*}, q^{*}: \Gamma^{*} \rightarrow G^{*}\right)
$$

called the dual of $T$, where $i_{\Gamma^{*}}=p_{A}, t_{\Gamma^{*}}=q_{A}, i_{G^{*}}=p_{V}$ and $t_{G^{*}}=q_{V}$.

Let $T=(p, q: \Gamma \rightarrow G)$ be a textile system. Let $\xi=\phi_{p}$ and let $\eta=\phi_{q}$. A two-dimensional configuration $\left(\alpha_{i j}\right)_{i, j \in \mathbf{Z}}, \alpha_{i j} \in A_{\Gamma}$, is called a textile woven by $T$ if $\left(\alpha_{i j}\right)_{j \in \mathbf{Z}} \in X_{\Gamma}$ and $\eta\left(\left(\alpha_{i-1, j}\right)_{j \in \mathbf{Z}}\right)=\xi\left(\left(\alpha_{i j}\right)_{j \in \mathbf{Z}}\right)$ for all $i \in \mathbf{Z}$. Let $U_{T}$ denote the set of all textiles woven by $T$. Define

$$
Z_{T}=\left\{\left(\alpha_{0 j}\right)_{j \in \mathbf{Z}} \mid \exists\left(\alpha_{i j}\right)_{i, j \in \mathbf{Z}} \in U_{T}\right\}, \quad X_{T}=\left\{\xi\left(\left(\alpha_{0 j}\right)_{j \in \mathbf{Z}}\right) \mid \exists\left(\alpha_{i j}\right)_{i, j \in \mathbf{Z}} \in U_{T}\right\} .
$$


Then we have subshifts $\left(Z_{T}, \varsigma_{T}\right)$ and $\left(X_{T}, \sigma_{T}\right)$. We call $\left(X_{T}, \sigma_{T}\right)$ the woof shift of $T$ and $\left(X_{T^{*}}, \sigma_{T^{*}}\right)$ the warp shift of $T$. We say that $T$ is nondegenerate if $\left(X_{T}, \sigma_{T}\right)=$ $\left(X_{G}, \sigma_{G}\right)$. We define onto maps $\xi_{T}: Z_{T} \rightarrow X_{T}$ and $\eta_{T}: Z_{T} \rightarrow X_{T}$ to be the restrictions of $\xi$ and $\eta$, respectively. If $T$ is onesided 1-1, i.e., $\xi_{T}$ is 1-1, then an onto endomorphism $\varphi_{T}$ of $\left(X_{T}, \sigma_{T}\right)$ is defined by

$$
\varphi_{T}=\eta_{T} \xi_{T}^{-1} \text {. }
$$

If $T$ is 1-1, i.e., both $\xi_{T}$ and $\eta_{T}$ are 1-1, then $\varphi_{T}$ is an automorphism of $\left(X_{T}, \sigma_{T}\right)$. We also have the onesided subshifts $\left(\tilde{Z}_{T}, \tilde{\varsigma}_{T}\right)$ and $\left(\tilde{X}_{T}, \tilde{\sigma}_{T}\right)$ induced by $\left(Z_{T}, \varsigma_{T}\right)$ and $\left(X_{T}, \sigma_{T}\right)$, respectively. We define maps $\tilde{\xi}_{T}: \tilde{Z}_{T} \rightarrow \tilde{X}_{T}$ and $\tilde{\eta}_{T}: \tilde{Z}_{T} \rightarrow \tilde{X}_{T}$ by the restrictions of $\tilde{\phi}_{p}$ and $\tilde{\phi}_{q}$, respectively. If $T$ is onesided 1-1, then an onto continuous map $\bar{\chi}_{T}: X_{T} \rightarrow \tilde{X}_{T^{*}}$ is defined by

$$
\bar{\chi}_{T}\left(\xi_{T}\left(\left(\alpha_{1 j}\right)_{j \in \mathbf{Z}}\right)\right)=\tilde{\xi}_{T^{*}}\left(\left(\alpha_{i 1}\right)_{i \in \mathbf{N}}\right), \quad\left(\alpha_{i j}\right)_{i, j \in \mathbf{Z}} \in U_{T}, \alpha_{i j} \in A_{\Gamma} .
$$

If both $\tilde{\xi}_{T^{*}}$ and $\tilde{\eta}_{T^{*}}$ are $1-1$, then an onto continuous map $\bar{\chi}_{T}^{*}: \tilde{X}_{T^{*}} \rightarrow X_{T}$ is defined by

$$
\bar{\chi}_{T}^{*}\left(\tilde{\xi}_{T^{*}}\left(\left(\alpha_{i 1}\right)_{i \in \mathbf{N}}\right)\right)=\xi_{T}\left(\left(\alpha_{1 j}\right)_{j \in \mathbf{Z}}\right), \quad\left(\alpha_{i j}\right)_{i, j \in \mathbf{Z}} \in U_{T}, \alpha_{i j} \in A_{\Gamma} .
$$

If $\tilde{\xi}_{T^{*}}$ is $1-1$, then an onto continuous map $\hat{\chi}_{T^{*}}: \tilde{X}_{T^{*}} \rightarrow \tilde{X}_{T}$ is defined by

$$
\hat{\chi}_{T^{*}}\left(\tilde{\xi}_{T^{*}}\left(\left(\alpha_{i 1}\right)_{i \in \mathbf{N}}\right)\right)=\tilde{\xi}_{T}\left(\left(\alpha_{1 j}\right)_{j \in \mathbf{N}}\right), \quad\left(\alpha_{i j}\right)_{i, j \in \mathbf{Z}} \in U_{T}, \alpha_{i j} \in A_{\Gamma} .
$$

Theorem 2.1 (Theorem 2.11 of [N2]). Let $T$ be a textile system.

(1) If $T$ is onesided 1-1, then $\varphi_{T}$ is positively expansive if and only if both $\tilde{\xi}_{T^{*}}$ and $\tilde{\eta}_{T^{*}}$ are $1-1$.

(2) If $\tilde{\xi}_{T^{*}}$ is 1-1, then $\tilde{\varphi}_{T^{*}}$ is an expansive continuous map if and only if $T$ is onesided 1-1.

(3) If $T$ is onesided 1-1 and both $\tilde{\xi}_{T^{*}}$ and $\tilde{\eta}_{T^{*}}$ are $1-1$, then $\bar{\chi}_{T}$ is a homeomorphism with $\bar{\chi}_{T}^{*}=\bar{\chi}_{T}^{-1}$ and not only $\left(X_{T}, \sigma_{T}\right)$ and $\left(\tilde{X}_{T^{*}}, \tilde{\varphi}_{T^{*}}\right)$ but also $\left(X_{T}, \varphi_{T}\right)$ and $\left(\tilde{X}_{T^{*}}, \tilde{\sigma}_{T^{*}}\right)$ are topologically conjugate through $\bar{\chi}_{T}$.

Let $G$ be a graph. For $n \geq 0$, let $L_{n}(G)$ denote the set of all paths of length $n$ in $G$ and let $L(G)$ be the set of all paths in $G$, that is,

$$
\begin{gathered}
L_{n}(G)=\left\{a_{1} \ldots a_{n} \mid a_{j} \in A_{G}, t_{G}\left(a_{j}\right)=i_{G}\left(a_{j+1}\right) \text { for } j=1, \ldots, n-1\right\} \quad \text { for } n \geq 1, \\
L_{0}(G)=V_{G} \quad \text { and } \quad L(G)=\bigcup_{n=0}^{\infty} L_{n}(G) .
\end{gathered}
$$

We extend $i_{G}: A_{G} \rightarrow V_{G}$ and $t_{G}: A_{G} \rightarrow V_{G}$ to $i_{G}: L(G) \rightarrow V_{G}$ and $t_{G}: L(G) \rightarrow$ $V_{G}$, respectively, as follows. Define $i_{G}(v)=t_{G}(v)=v$ for $v \in V_{G}$. If $n \geq 1$, then for $w=a_{1} \ldots a_{n} \in L_{n}(G)$ with $a_{j} \in A_{G}$, we define $i_{G}(w)=i_{G}\left(a_{1}\right)$ and $t_{G}(w)=t_{G}\left(a_{n}\right)$. We also extend $h_{V}$ and $h_{A}$ of a graph-homomorphism $h: \Gamma \rightarrow G$ to $h: L(\Gamma) \rightarrow L(G)$ as follows. We define $h(\bar{v})=h_{V}(\bar{v})$ for $\bar{v} \in V_{\Gamma}$. If $n \geq 1$, then for $\bar{w}=\alpha_{1} \ldots \alpha_{n} \in L_{n}(\Gamma)$ with $\alpha_{j} \in A_{\Gamma}$, we define $h(\bar{w})=h_{A}\left(\alpha_{1}\right) \ldots h_{A}\left(\alpha_{n}\right)$.

For $n \geq 2$, let $G^{[n]}$ be the graph defined as follows: $A_{G^{[n]}}=L_{n}(G), V_{G^{[n]}}=$ $L_{n-1}(G)$, and $i_{G^{[n]}}$ and $t_{G^{[n]}}$ are the mappings such that for $w=a_{1} \ldots a_{n} \in A_{G^{[n]}}$ with $a_{j} \in A_{G}, i_{G^{[n]}}(w)=a_{1} \ldots a_{n-1}$ and $t_{G^{[n]}}(w)=a_{2} \ldots a_{n}$. We define $G^{[1]}=G$. We call $G^{[n]}$ the higher block graph of order $n$ of $G$. For a graph-homomorphism $h: \Gamma \rightarrow G$ and $n \geq 1$, we define the higher block homomorphism of order $n$ of $h$, written $h^{[n]}: \Gamma^{[n]} \rightarrow G^{[n]}$, by $h_{A}^{[n]}(w)=h(w), w \in L_{n}(\Gamma)$. For $n \geq 1$ the $n$-th 
power $G^{n}$ of $G$ is defined to be the graph such that $A_{G^{n}}=L_{n}(G), V_{G^{n}}=V_{G}$ and $i_{G^{n}}(w)=i_{G}(w)$ and $t_{G^{n}}(w)=t_{G}(w)$ for $w \in L_{n}(G)$. For a graph-homomorphism $h: \Gamma \rightarrow G$ and $n \geq 1$, we define the $n$-th product-power $h^{(n)}: \Gamma^{n} \rightarrow G^{n}$ of $h$ by $h_{A}^{(n)}(w)=h(w), w \in L_{n}(G)$.

For a textile system $T=(p, q: \Gamma \rightarrow G)$ such that the following definitions are well defined (see [N2, pp. 17, 18]) and for $n \geq 1$, we define the higher block system $T^{[n]}$ of order $n$ of $T$ by $T^{[n]}=\left(p^{[n]}, q^{[n]}: \Gamma^{[n]} \rightarrow G^{[n]}\right)$, define the $n$-th product-power $T^{(n)}$ of $T$ by $T^{(n)}=\left(p^{(n)}, q^{(n)}: \Gamma^{n} \rightarrow G^{n}\right)$, and define the $n$-th composition-power $T^{n}$ of $T$ by $T^{n}=\left(\left(T^{*}\right)^{(n)}\right)^{*}$.

\section{Nondegenerate $q$-BIRESOlving TeXtile Systems}

A graph $G$ is said to be nondegenerate if both $i_{G}$ and $t_{G}$ are onto. A graphhomomorphism $h: \Gamma \rightarrow G$ is said to be onto if both $h_{A}$ and $h_{V}$ are onto.

An onto graph-homomorphism $h: \Gamma \rightarrow G$ between nondegenerate graphs is said to be right resolving if for each $u \in V_{\Gamma}$, the restriction of $h_{A}$ on $i_{\Gamma}^{-1}(\{u\})$ is a bijection onto $i_{G}^{-1}\left(\left\{h_{V}(u)\right\}\right)$. It is said to be left resolving if for each $u \in V_{\Gamma}$, the restriction of $h_{A}$ on $t_{\Gamma}^{-1}(\{u\})$ is a bijection onto $t_{G}^{-1}\left(\left\{h_{V}(u)\right\}\right)$.

A textile system $T=(p, q: \Gamma \rightarrow G)$ is said to be $q$-biresolving if $q$ is both left resolving and right resolving. It is said to be $L L$ if both $p$ and $q$ are left resolving, or equivalently $T^{*}$ is $q$-biresolving.

For a graph $G$, let $M_{G}$ denote its adjacency matrix, i.e., $M_{G}=\left(M_{G}(u, v)\right)_{u, v \in V_{G}}$, where $M_{G}(u, v)$ is the number of arcs starting from vertex $u$ and ending in vertex $v$. Let $\lambda_{G}$ denote the spectral radius of $M_{G}$.

Proposition 3.1. Let $T=(p, q: \Gamma \rightarrow G)$ be a (nondegenerate) $q$-biresolving textile system with $G$ irreducible, $\phi_{p}$ bounded-to-one and $\phi_{q} N$-to-one.

(1) If $(l(u))_{u \in V_{G}}$ and $(r(u))_{u \in V_{G}}$ are left and right eigenvectors of $M_{G}$ corresponding to $\lambda_{G}$, then $(1, \ldots, 1)$ indexed by $V_{G}$ and $(l(u) r(u))_{u \in V_{G}}$ are left and right eigenvectors of $M_{G^{*}}$ corresponding to $\lambda_{G^{*}}=N$, where $G^{*}$ is the graph over which $T^{*}$ is defined.

(2) If $\sigma_{\left(T^{[k]}\right) *}$ is topologically transitive for all $k \geq 1$ and $G$ is aperiodic, then $\lambda_{G}$ is a rational integer.

(3) If $T$ is onesided 1-1 and $\sigma_{T^{*}}$ is topologically transitive, then $\bar{\chi}_{T}$ is measure preserving with respect to the Parry measures.

(4) If $T^{*}$ is $1-1$ and $\sigma_{T^{*}}$ is topologically transitive, then $\bar{\chi}_{T}^{*}$ is measure preserving with respect to the Parry measures.

(5) If $T^{*}$ is onesided 1-1 and $\sigma_{T^{*}}$ is topologically transitive, then $\hat{\chi}_{T^{*}}$ is measure preserving with respect to the Parry measures.

To prove Proposition 3.1, we need a lemma, which should be compared with $\mathrm{B}$. Lemma 2.1] and [H], Theorem 5.4].

Let $h: \Gamma \rightarrow G$ be a graph-homomorphism. Let $w \in L(G)$. Let $M_{G}(w)$ be the matrix $\left(M_{G}(w)(u, v)\right)_{u, v \in V_{G}}$ defined as follows. If $w \in V_{G}$, then $M_{G}(w)(u, v)=1$ if $u=v=w$, and otherwise $M_{G}(w)(u, v)=0$. If $w \in L_{n}(G)$ with $n \geq 1$, then $M_{G}(w)(u, v)=1$ if $i_{G}(w)=u$ and $t_{G}(w)=v$, and otherwise $M_{G}(w)(u, v)=0$. Define $M_{h}(w)$ to be the matrix $\left(M_{h}(w)(\bar{u}, \bar{v})\right)_{\bar{u}, \bar{v} \in V_{\Gamma}}$ such that if $w \in V_{G}$, then $M_{h}(w)(\bar{u}, \bar{v})=1$ if $\bar{u}=\bar{v}$ and $h_{V}(\bar{u})=w$, and otherwise $M_{h}(w)(\bar{u}, \bar{v})=0$. If $w \in L_{n}(G)$ with $n \geq 1$, then $M_{h}(w)(\bar{u}, \bar{v})$ is the number of paths $\bar{w} \in L_{n}(\Gamma)$ such that $i_{\Gamma}(\bar{w})=\bar{u}, t_{\Gamma}(\bar{w})=\bar{v}$ and $h(\bar{w})=w$. 
Lemma 3.2. If $h: \Gamma \rightarrow G$ is a graph-homomorphism between irreducible graphs with $\lambda_{G}=\lambda_{\Gamma}=\lambda$ such that the 1-block map $\phi_{h}$ given by $h$ is onto, then

$$
\frac{\sum_{\bar{w} \in h^{-1}(\{w\})} \bar{l}\left(i_{\Gamma}(\bar{w})\right) \bar{r}\left(t_{\Gamma}(\bar{w})\right)}{l\left(i_{G}(w)\right) r\left(t_{G}(w)\right)}=\frac{\bar{l} \bar{r}}{l r} \quad \forall w \in L(G),
$$

where $l=(l(u))_{u \in V_{G}}$ and $r=(r(u))_{u \in V_{G}}$ are left and right eigenvectors of $M_{G}$ corresponding to $\lambda$, and $\bar{l}=(\bar{l}(\bar{u}))_{\bar{u} \in V_{\Gamma}}$ and $\bar{r}=(\bar{r}(\bar{u}))_{\bar{u} \in V_{\Gamma}}$ are those of $M_{\Gamma}$ corresponding to $\lambda$.

Proof. We use the extremal argument of Boyle [B].

Since $G$ and $\Gamma$ are irreducible graphs with the same spectral radius and $\phi_{h}$ is onto, $h: L(\Gamma) \rightarrow L(G)$ is bounded-to-one and onto ([तP $)$. Therefore it follows that

$$
\left\{\frac{\bar{l} M_{h}(w) \bar{r}}{l M_{G}(w) r} \mid w \in L(G)\right\}
$$

is a finite set of positive numbers. Let $s$ and $t$ be respectively the minimum and maximum numbers among them. Let $w_{1} \in L(G)$ be such that $\left(\bar{l} M_{h}\left(w_{1}\right) \bar{r}\right) /\left(l M_{G}\left(w_{1}\right) r\right)$ $=s$. We claim that

$$
\frac{\bar{l} M_{h}\left(x w_{1}\right) \bar{r}}{l M_{G}\left(x w_{1}\right) r}=s \quad \forall x \in L(G) \text { with } x w_{1} \in L(G) .
$$

To prove this, let $L_{1}=\left\{x \in L_{k}(G) \mid t_{G}(x)=i_{G}\left(w_{1}\right)\right\}$ with $k \geq 0$. Then

$$
\begin{aligned}
s & =\frac{\lambda^{k} \bar{l} M_{h}\left(w_{1}\right) \bar{r}}{\lambda^{k} l M_{G}\left(w_{1}\right) r}=\frac{\bar{l} M_{\Gamma}^{k} M_{h}\left(w_{1}\right) \bar{r}}{l M_{G}^{k} M_{G}\left(w_{1}\right) r}=\frac{\bar{l}\left(\sum_{x \in L_{k}(G)} M_{h}(x)\right) M_{h}\left(w_{1}\right) \bar{r}}{l\left(\sum_{x \in L_{k}(G)} M_{G}(x)\right) M_{G}\left(w_{1}\right) r} \\
& =\frac{\sum_{x \in L_{1}} \bar{l} M_{h}\left(x w_{1}\right) \bar{r}}{\sum_{x \in L_{1}} l M_{G}\left(x w_{1}\right) r} .
\end{aligned}
$$

Since $\left(\bar{l} M_{h}\left(x w_{1}\right) \bar{r}\right) /\left(l M_{G}\left(x w_{1}\right) r\right) \geq s$ for all $x \in L_{1}$, if there were $x \in L_{1}$ with $\left(\bar{l} M_{h}\left(x w_{1}\right) \bar{r}\right) /\left(l M_{G}\left(x w_{1}\right) r\right)>s$, then it would follow that

$$
\frac{\sum_{x \in L_{1}} \bar{l} M_{h}\left(x w_{1}\right) \bar{r}}{\sum_{x \in L_{1}} l M_{G}\left(x w_{1}\right) r}>s .
$$

Hence the claim is proved. Similarly we see that if $w_{2} \in L(G)$ is such that $\left(\bar{l} M_{h}\left(w_{2}\right) \bar{r}\right) /\left(l M_{G}\left(w_{2}\right) r\right)=t$, then

$$
\frac{\bar{l} M_{h}\left(w_{2} y\right) \bar{r}}{l M_{G}\left(w_{2} y\right) r}=t \quad \forall y \in L(G) \text { with } w_{2} y \in L(G) .
$$

Since $G$ is irreducible, there is a path $z \in L(G)$ such that $w_{2} z w_{1} \in L(G)$. By the above we have

$$
s=\frac{\bar{l} M_{h}\left(w_{2} z w_{1}\right) \bar{r}}{l M_{G}\left(w_{2} z w_{1}\right) r}=t .
$$

Therefore, for any $w \in L(G)$ and any $u \in V_{G}$, we have

$$
\frac{\bar{l} M_{h}(w) \bar{r}}{l M_{G}(w) r}=\frac{\bar{l} M_{h}(u) \bar{r}}{l M_{G}(u) r}=\frac{\sum_{u \in V_{G}} \bar{l} M_{h}(u) \bar{r}}{\sum_{u \in V_{G}} l M_{G}(u) r}=\frac{\bar{l} \bar{r}}{l r} .
$$


Proof of Proposition 3.1. (1) Since $q$ is biresolving with $\phi_{q} N$-to-one, $q_{V}$ is $N$-toone and onto. Hence in $G^{*}$ every vertex has exactly $N$ arcs ending in it, so that $(1, \ldots, 1)$ indexed by $V_{G}\left(=V_{G^{*}}\right)$ is a left eigenvector of $M_{G^{*}}$ corresponding to $\lambda_{G^{*}}=N$. Since $q: \Gamma \rightarrow G$ is biresolving with $G$ irreducible, $\Gamma$ is the disjoint union of finitely many irreducible graphs, say $\Gamma_{i}$ 's, with $\lambda_{\Gamma_{i}}=\lambda_{G}$ for all $i$. Since $\phi_{p}$ is bounded-to-one, if $p_{i}: \Gamma_{i} \rightarrow G$ is the restriction of $p$, then $\phi_{p_{i}}$ is bounded-to-one and onto. From [N1, Proposition 2.3], it follows that the row and column vectors $\bar{l}=\left(l\left(q_{V}(\bar{u})\right)\right)_{\bar{u} \in V_{\Gamma}}$ and $\bar{r}=\left(r\left(q_{V}(\bar{u})\right)\right)_{\bar{u} \in V_{\Gamma}}$ are left and right eigenvectors of $M_{\Gamma}$ corresponding to $\lambda_{\Gamma}\left(=\lambda_{G}\right)$. Therefore, applying Lemma 3.2 to every $p_{i}$, we have

$$
\frac{\sum_{\bar{u} \in p_{V}^{-1}(\{u\})} l\left(q_{V}(\bar{u})\right) r\left(q_{V}(\bar{u})\right)}{l(u) r(u)}=N \quad \forall u \in V_{G} .
$$

This implies that the strictly positive column vector $(l(u) r(u))_{u \in V_{G}}$ is a right eigenvector of $M_{G^{*}}$ corresponding to $\lambda_{G^{*}}=N$.

(2) For $k \geq 1, V_{G^{[k]}}=L_{k-1}(G)$, and $\left(l\left(i_{G}(w)\right)\right)_{w \in V_{G^{[k]}}}$ and $\left(r\left(t_{G}(w)\right)\right)_{w \in V_{G^{[k]}}}$ are the left and right eigenvectors of $M_{G^{[k]}}$ corresponding to $\lambda_{G^{[k]}}=\lambda_{G}=N$. Hence applying the preceding equation (for $T$ ) to $T^{[k]}$ for every $k \geq 1$, we have

$$
\frac{\sum_{\bar{w} \in p^{-1}(\{w\})} l\left(i_{G}(q(\bar{w}))\right) r\left(t_{G}(q(\bar{w}))\right)}{l\left(i_{G}(w)\right) r\left(t_{G}(w)\right)}=N \quad \forall w \in L(G),
$$

since for $h=p, q,\left(h^{[k]}\right)_{V}(\bar{w})=h(\bar{w})$ for $\bar{w} \in V_{\Gamma^{[k]}}=L_{k-1}(\Gamma)$. Let $G_{k}^{*}$ be the graph over which the textile system $\left(T^{[k]}\right)^{*}$ is defined. Then $A_{G_{k}^{*}}=L_{k-1}(\Gamma), V_{G_{k}^{*}}=$ $L_{k-1}(G)$ and for $\bar{w} \in A_{G_{k}^{*}}, i_{G_{k}^{*}}(\bar{w})=p(\bar{w})$ and $t_{G_{k}^{*}}(\bar{w})=q(\bar{w})$. Hence (3.1) implies that the strictly positive column vector $\left(l\left(i_{G}(w)\right) r\left(t_{G}(w)\right)\right)_{w \in L_{k-1}(G)}$ is a right eigenvector of $M_{G_{k}^{*}}$ corresponding to $\lambda_{G_{k}^{*}}=N$. Since $T^{[k]}$ is $q$-biresolving, $\left(T^{[k]}\right)^{*}$ is LL, so that $\left(T^{[k]}\right)^{*}$ is nondegenerate. Therefore, since $\sigma_{\left(T^{[k]}\right)^{*}}$ is transitive for all $k \geq 1, G_{k}^{*}$ is irreducible for all $k \geq 1$. Hence for all $k \geq 1$ the eigenvector $\left(l\left(i_{G}(w)\right) r\left(t_{G}(w)\right)\right)_{w \in L_{k-1}(G)}$ is a constant multiple of some column vector with all components in $\mathbf{Q}$. Let $u_{0} \in V_{G}$. Since $G$ is irreducible and aperiodic, there exists $k \geq 1$ such that for every $u \in V_{G}$, there exists a path $w \in L_{k-1}(G)$ with $i_{G}(w)=u_{0}$ and $t_{G}(w)=u$. Therefore the column vector $\left(l\left(u_{0}\right) r(u)\right)_{u \in V_{G}}$ is a constant multiple of a column vector with all components in $\mathbf{Q}$. Thus $\lambda_{G} \in \mathbf{Q}$. Since $\lambda_{G}$ is an algebraic integer, it is a rational integer.

Before proving (3), (4) and (5), we make some preliminary arguments.

Let $s \geq-1, s^{\prime} \geq 1$ and $t \geq 1$ be integers. A finite configuration of the form $\left(\alpha_{i j}\right)_{1 \leq i \leq t,-s \leq j \leq s^{\prime}}$ with $\alpha_{i, j} \in A_{\Gamma}$ is called a cloth of size $t \times\left(s+s^{\prime}+1\right)$ (woven by $T$ ), if $\alpha_{i,-s} \ldots \alpha_{i, s^{\prime}} \in L_{s+s^{\prime}+1}(\Gamma)$ for $1 \leq i \leq t$ and $q\left(\alpha_{i,-s} \ldots \alpha_{i, s^{\prime}}\right)=$ $p\left(\alpha_{i+1,-s} \ldots \alpha_{i+1, s^{\prime}}\right)$ for all $1 \leq i \leq t-1$. For a cloth $c=\left(\alpha_{i j}\right)_{1 \leq i \leq t,-s \leq j \leq s^{\prime}}$, its uppermost $G$-path $p\left(\alpha_{1,-s} \ldots \alpha_{1, s^{\prime}}\right)$, its lowermost $G$-path $q\left(\alpha_{t, 1} \ldots \alpha_{t, s^{\prime}}\right)$ and its axis $G^{*}$-path $\left(i_{\Gamma}\left(\alpha_{i, 1}\right)\right)_{1 \leq i \leq t}$ are denoted by $\mathrm{p}(c), \mathrm{q}(c)$ and $\mathrm{o}^{*}(c)$, respectively. Let $C_{t, s, s^{\prime}}$ denote the set of all cloths of size $t \times\left(s+s^{\prime}+1\right)$. Since $q$ is biresolving, $c \in C_{t, s, s^{\prime}}$ is uniquely determined by $\mathrm{q}(c)$ and $\mathrm{o}^{*}(c)$.

If $x \in L_{s+s^{\prime}+1}(G)$ and $C(x)=\left\{c \in C_{t, s, s^{\prime}} \mid \mathrm{p}(c)=x\right\}$, then by (3.1) we have

$$
\frac{\sum_{c \in C(x)} l\left(i_{G}(\mathrm{q}(c))\right) r\left(t_{G}(\mathrm{q}(c))\right)}{l\left(i_{G}(x)\right) r\left(t_{G}(x)\right)}=N^{t} .
$$

For $x \in L_{s+s^{\prime}+1}(G)$ and $y \in L_{t}\left(G^{*}\right)$, let $\Delta(x, y)=\{\mathrm{q}(c) \mid c \in C(x, y)\}$ with $C(x, y)=\left\{c \mid c \in C_{t, s, s^{\prime}}, \mathrm{p}(c)=x, \mathrm{o}^{*}(c)=y\right\}$. Then since $\mathrm{q}(c) \in \Delta(x, y)$ uniquely 
determines $c \in C(x, y)$, it follows that

$$
\frac{\sum_{y \in L_{t}\left(G^{*}\right)} \sum_{w \in \Delta(x, y)} l\left(i_{G}(w)\right) r\left(t_{G}(w)\right)}{l\left(i_{G}(x)\right) r\left(t_{G}(x)\right)}=N^{t} \quad \text { for } x \in L_{s+s^{\prime}+1}(G) .
$$

For $y \in L_{t}\left(G^{*}\right)$, let $\Delta(y)=\left\{a_{-s} \ldots a_{s^{\prime}} \in L_{s+s^{\prime}+1}(G) \mid a_{j} \in A_{G}, i_{G}\left(a_{1}\right)=t_{G^{*}}(y)\right\}$. Let $\Delta_{1}(y)$ be the set of all paths $w_{1} \in L_{s+1}(G)$ with $t_{G}\left(w_{1}\right)=t_{G^{*}}(y)$ and let $\Delta_{2}(y)$ be the set of all paths $w_{2} \in L_{s^{\prime}}(G)$ with $i_{G}\left(w_{2}\right)=t_{G^{*}}(y)$. Then $\Delta(y)=\left\{w_{1} w_{2} \mid\right.$ $\left.w_{1} \in \Delta_{1}(y), w_{2} \in \Delta_{2}(y)\right\}$. Thus

$$
\begin{gathered}
\sum_{w \in \Delta(y)} l\left(i_{G}(w)\right) r\left(t_{G}(w)\right)=\left(\sum_{w_{1} \in \Delta_{1}(y)} l\left(i_{G}\left(w_{1}\right)\right)\right)\left(\sum_{w_{2} \in \Delta_{2}(y)} r\left(t_{G}\left(w_{2}\right)\right)\right) \\
=\lambda_{G}^{s+1} l\left(t_{G}\left(w_{1}\right)\right) \lambda_{G}^{s^{\prime}} r\left(i_{G}\left(w_{2}\right)\right)=\lambda_{G}^{s+s^{\prime}+1} l\left(t_{G^{*}}(y)\right) r\left(t_{G^{*}}(y)\right) .
\end{gathered}
$$

(3) Let $y \in L_{t}\left(G^{*}\right)$ with $t \geq 1$. Since $\bar{\chi}_{T}$ is continuous, for the clopen set

$$
[y]=\left\{\left(b_{i}\right)_{i \in \mathbf{N}} \in \tilde{X}_{G^{*}} \mid b_{1} \ldots b_{t}=y\right\},
$$

there exist $s \geq 0, s^{\prime} \geq 1$ and distinct $x_{1}, \ldots, x_{m} \in L_{s+s^{\prime}+1}(G)$ such that

$$
\bar{\chi}_{T}^{-1}([y])=\left[-s x_{1}\right] \cup \cdots \cup\left[-s x_{m}\right],
$$

where for $x \in L_{s+s^{\prime}+1}(G),[-s \quad x]$ denotes the cylinder set $\left\{\left(a_{j}\right)_{j \in \mathbf{Z}} \in X_{G}\right.$ $\left.a_{-s} \ldots a_{s^{\prime}}=x\right\}$. Since $q$ is biresolving, for any $w \in \Delta(y)$ there exists a unique $c \in C_{t, s, s^{\prime}}$ such that $\mathrm{q}(c)=w$ and $\mathrm{o}^{*}(c)=y$, and for any such $c, \mathrm{p}(c) \in\left\{x_{1}, \ldots, x_{m}\right\}$ because $c$ can be extended to a textile (because $q$ is biresolving and $T$ is nondegenerate). Therefore, we have $\Delta(y)=\bigcup_{k=1}^{m} \Delta\left(x_{k}, y\right)$ with $\Delta\left(x_{k}, y\right) \cap \Delta\left(x_{k^{\prime}}, y\right)=\emptyset$, $1 \leq k<k^{\prime} \leq m$. Thus using (3.2) we have

$$
\begin{aligned}
\sum_{w \in \Delta(y)} l\left(i_{G}(w)\right) r\left(t_{G}(w)\right) & =\sum_{k=1}^{m} \sum_{w \in \Delta\left(x_{k}, y\right)} l\left(i_{G}(w)\right) r\left(t_{G}(w)\right) \\
& =\sum_{k=1}^{m} N^{t} l\left(i_{G}\left(x_{k}\right)\right) r\left(t_{G}\left(x_{k}\right)\right) .
\end{aligned}
$$

Hence by (3.3) we have

$$
\frac{l\left(t_{G^{*}}(y)\right) r\left(t_{G^{*}}(y)\right)}{N^{t}}=\frac{\sum_{k=1}^{m} l\left(i_{G}\left(x_{k}\right)\right) r\left(t_{G}\left(x_{k}\right)\right)}{\lambda_{G}^{s+s^{\prime}+1}} .
$$

Since $G$ and $G^{*}$ are irreducible, the Parry measures $\mu_{G}$ and $\tilde{\mu}_{G^{*}}$ can be considered for $\sigma_{G}$ and $\tilde{\sigma}_{G^{*}}$. The above implies that

$$
\tilde{\mu}_{G^{*}}([y])=\sum_{k=1}^{m} \mu_{G}\left(\left[-s x_{k}\right]\right)=\mu_{G}\left(\bar{\chi}_{T}^{-1}([y])\right) .
$$

Thus $\bar{\chi}_{T}$ is measure preserving with respect to the Parry measures.

(4) Let $x \in L_{s+s^{\prime}+1}(G)$ with $s \geq 0$ and $s^{\prime} \geq 1$. Since $\bar{\chi}_{T}^{*}$ is continuous, there exist $t \geq 1$ and distinct $y_{1}, \ldots, y_{n} \in L_{t}\left(G^{*}\right)$ such that

$$
\left(\bar{\chi}_{T}^{*}\right)^{-1}\left(\left[{ }_{-s} x\right]\right)=\left[y_{1}\right] \cup \cdots \cup\left[y_{n}\right] .
$$


Since $\Delta\left(x, y_{k}\right)=\Delta\left(y_{k}\right)$ for $1 \leq k \leq n$, using (3.2) and (3.3) we have

$$
\begin{aligned}
N^{t} l\left(i_{G}(x)\right) r\left(t_{G}(x)\right) & =\sum_{k=1}^{n} \sum_{w \in \Delta\left(x, y_{k}\right)} l\left(i_{G}(w)\right) r\left(t_{G}(w)\right)=\sum_{k=1}^{n} \sum_{w \in \Delta\left(y_{k}\right)} l\left(i_{G}(w)\right) r\left(t_{G}(w)\right) \\
& =\sum_{k=1}^{n} \lambda_{G}^{s+s^{\prime}+1} l\left(t_{G^{*}}\left(y_{k}\right)\right) r\left(t_{G^{*}}\left(y_{k}\right)\right)
\end{aligned}
$$

so that we have

$$
\frac{l\left(i_{G}(x)\right) r\left(t_{G}(x)\right)}{\lambda_{G}^{s+s^{\prime}+1}}=\frac{\sum_{k=1}^{n} l\left(t_{G^{*}}\left(y_{k}\right)\right) r\left(t_{G^{*}}\left(y_{k}\right)\right)}{N^{t}} .
$$

Thus we have

$$
\mu_{G}([-s x])=\sum_{k=1}^{n} \tilde{\mu}_{G^{*}}\left(\left[y_{k}\right]\right)=\tilde{\mu}_{G^{*}}\left(\left(\bar{\chi}_{T}^{*}\right)^{-1}([-s x])\right) .
$$

(5) Let $x \in L_{s^{\prime}}(G)$ with $s^{\prime} \geq 1$. Since $\hat{\chi}_{T^{*}}$ is continuous, there exist $t \geq 1$ and distinct $y_{1}, \ldots, y_{n} \in L_{t}\left(G^{*}\right)$ such that

$$
\left(\hat{\chi}_{T^{*}}\right)^{-1}([x])=\left[y_{1}\right] \cup \cdots \cup\left[y_{n}\right] .
$$

If we consider the argument in the proof of (4) for the case that $s=-1$, we see that

$$
\frac{l\left(i_{G}(x)\right) r\left(t_{G}(x)\right)}{\lambda_{G}^{s^{\prime}}}=\frac{\sum_{k=1}^{n} l\left(t_{G^{*}}\left(y_{k}\right)\right) r\left(t_{G^{*}}\left(y_{k}\right)\right)}{N^{t}} .
$$

Thus we have

$$
\tilde{\mu}_{G}([x])=\sum_{k=1}^{n} \tilde{\mu}_{G^{*}}\left(\left[y_{k}\right]\right)=\tilde{\mu}_{G^{*}}\left(\left(\bar{\chi}_{T}^{*}\right)^{-1}([x])\right) .
$$

We remark that (3) ((4)) of Proposition 3.1 combined with Theorem 4.1 (in Section 4) and [Wa, the proof of Theorem 8.8] give another proof of the result of Boyle and Maass [BM]: if $\varphi$ is a positively expansive endomorphism of a mixing topological Markov shift $(X, \sigma)$, then $\sigma$ and $\varphi$ have the same measure of maximal entropy.

Corollary 3.3. Let $T=(p, q: \Gamma \rightarrow G)$ be a (nondegenerate) $q$-biresolving textile system with $G$ irreducible and aperiodic, $\phi_{p}$ bounded-to-one and $\phi_{q} N$-to-one.

(1) If $T$ is onesided 1-1 and $\varphi_{T}$ is topologically transitive, then $\lambda_{G}$ is an integer, say $J$, and every prime dividing $N$ divides $J$.

(2) If $T^{*}$ is onesided 1-1, then $\lambda_{G}$ is an integer, say $J$, and every prime dividing $J$ divides $N$.

(3) If both $T$ and $T^{*}$ are onesided 1-1, then $\lambda_{G}$ is an integer, say $J$, and $J$ and $N$ are divisible by the same primes.

Proof. (1) Since $\varphi_{T}$ is topologically transitive, it follows that $\sigma_{\left(T^{[k]}\right]^{*}}$ is topologically transitive for all $k \geq 1$. Hence $\lambda_{G}$ is an integer, say $J$, by (2) of Proposition 3.1. Thus we may assume that all the components of $(l(u))_{u \in V_{G}}$ and $(r(u))_{u \in V_{G}}$ in the proposition are integers. By (3.4) we have

$$
J^{s+s^{\prime}+1} l\left(t_{G^{*}}(y)\right) r\left(t_{G^{*}}(y)\right)=N^{t} \sum_{k=1}^{m} l\left(i_{G}\left(x_{k}\right)\right) r\left(t_{G}\left(x_{k}\right)\right) .
$$


Since the length $t$ of $y$ can be arbitrarily large without changing $l\left(t_{G^{*}}(y)\right) r\left(t_{G^{*}}(y)\right)$, every prime dividing $N$ must divide $J$.

(2) It follows from [N4, Proposition 5.1] that $\left(X_{T^{*}}, \sigma_{T^{*}}\right)$ is conjugate to a full shift. Since $T^{*}$ is onesided $1-1,\left(X_{\left(T^{[k]}\right)^{*}}, \sigma_{\left(T^{[k]}\right)^{*}}\right)$ and $\left(X_{T^{*}}, \sigma_{T^{*}}\right)$ are topologically conjugate for all $k \geq 1$, so that $\sigma_{\left(T^{[k]}\right)^{*}}$ is topologically transitive for all $k \geq 1$. Thus (2) is proved by a discussion similar to that in (1) by using (3.5).

(3) By the proofs of (1) and (2), (3) is proved.

We also remark that Corollary 3.3(3) combined with Theorem 4.1 (in Section 4) recovers a small part of the result of Boyle and Maass [BM] stated in the Introduction.

Marcus proved in $[\mathrm{M}]$ the theorem that if $G$ is an irreducible graph whose spectral radius is an integer $J$, then there exists an irreducible graph $G^{\prime}$ such that $\left(X_{G}, \sigma_{G}\right)$ and $\left(X_{G^{\prime}}, \sigma_{G^{\prime}}\right)$ are topologically conjugate, each row sum of $M_{G^{\prime}}$ is $J$ and each column sum of $M_{G^{\prime}}$ is $J$. His theorem and his proof are the right things to make use of in order to prove the third conjecture of Boyle and Maass in [BM]. His proof will be described in a way suitable to our needs.

For a mapping $f: A \rightarrow B$ between finite sets, we define a (rectangular) matrix $M_{f}=\left(M_{f}(u, v)\right)_{u \in A, v \in B}$ such that $M_{f}(u, v)=1$ if $f(u)=v$, and otherwise $M_{f}(u, v)=0$. For a matrix $M, M^{t}$ denotes its transpose. Hence, for example, $M_{G}=M_{i_{G}}^{t} M_{t_{G}}$ for a graph $G$.

Let $G$ be a nondegenerate graph. Let $\stackrel{e}{\sim}$ be an equivalence relation on $A_{G}$ such that for $a_{1}, a_{2} \in A_{G}$, if $a_{1} \stackrel{e}{\sim} a_{2}$, then $i_{G}\left(a_{1}\right)=i_{G}\left(a_{2}\right)$, where $e$ denotes the canonical map of $\stackrel{e}{\sim}$ which maps each $a \in A_{G}$ to the equivalence class $e(a)$ containing $a$. Let $C_{e}$ denote the set of equivalence classes of $\stackrel{e}{\sim}$. Let $\bar{C}_{e}=\left\{\bar{e}(a) \mid a \in A_{G}\right\}$, where $\bar{e}(a)$ is merely a copy of $e(a)$. Let $H_{e}$ be a bipartite graph defined as follows. Let $A_{H_{e}}=A_{G} \cup \bar{C}_{e}$ and let $V_{H_{e}}=V_{G} \cup C_{e}$. We define $i_{H_{e}}$ and $t_{H_{e}}$ by

$$
i_{G}(a) \stackrel{i_{H_{e}}}{\longleftarrow} \bar{e}(a) \stackrel{t_{H_{e}}}{\longmapsto} e(a), \quad e(a) \stackrel{i_{H_{e}}}{\longleftarrow} a \stackrel{t_{H_{e}}}{\longmapsto} t_{G}(a), \quad a \in A_{G} .
$$

Let $L_{e}=M_{e}^{t}$. We define $\breve{e}: C_{e} \rightarrow V_{G}$ by $e(a) \mapsto i_{G}(a)$. Let $L_{\breve{e}}=M_{\breve{e}}^{t}$. Let $R=M_{t_{G}}$. Then $M_{H_{e}}$ is written in the form

$$
M_{H_{e}}=\left(\begin{array}{cc}
0 & L_{\breve{e}} \\
L_{e} R & 0
\end{array}\right)
$$

where the indexing set is $V_{G} \cup C_{e}$. The bipartite graph $H_{e}$ induces a pair of graphs $\dot{H}_{e}$ and $\grave{H}_{e}$ such that the arcs of $\dot{H}_{e}$ are the paths of length 2 in $H_{e}$ each starting from a vertex in $V_{G}$ and ending in a vertex in $V_{G}$, and the arcs of $\grave{H}_{e}$ are the paths of length 2 in $H_{e}$ starting from a vertex in $C_{e}$ and ending in a vertex in $C_{e}$. There exists a graph-isomorphism $k_{e}: G \rightarrow \dot{H}_{e}$ with $\left(k_{e}\right)_{A}: a \mapsto \bar{e}(a) a, a \in A_{G}$. There exists a "bipartite" conjugacy $\zeta_{e}:\left(X_{\dot{H}_{e}}, \sigma_{\dot{H}_{e}}\right) \rightarrow\left(X_{\grave{H}_{e}}, \sigma_{\grave{H}_{e}}\right)$ defined by

$$
\left(\bar{e}\left(a_{j}\right) a_{j}\right)_{j \in \mathbf{Z}} \stackrel{\zeta_{e}}{\mapsto}\left(a_{j} \bar{e}\left(a_{j+1}\right)\right)_{j \in \mathbf{Z}}, \quad\left(a_{j}\right)_{j \in \mathbf{Z}} \in X_{G} .
$$

Define $G_{e}=\grave{H}_{e}$. Define $\psi_{e}=\zeta_{e} \kappa_{e}$, where $\kappa_{e}$ is the "symbolic" conjugacy given by $\kappa_{e}=\phi_{k_{e}}$. Then

(i) $\psi_{e}$ is a conjugacy (a "bipartite code") of $\left(X_{G}, \sigma_{G}\right)$ onto $\left(X_{G_{e}}, \sigma_{G_{e}}\right)$. 
We have

$$
\left(\begin{array}{cc}
M_{G} & 0 \\
0 & M_{G_{e}}
\end{array}\right)=\left(\begin{array}{cc}
M_{\dot{H}_{e}} & 0 \\
0 & M_{\grave{H}_{e}}
\end{array}\right)=\left(M_{H_{e}}\right)^{2}=\left(\begin{array}{cc}
L_{\breve{e}} L_{e} R & 0 \\
0 & L_{e} R L_{\breve{e}}
\end{array}\right),
$$

so that we have $M_{G}=L_{\breve{e}} L_{e} R$ and $M_{G_{e}}=L_{e} R L_{\breve{e}}$. Since $(1, \ldots, 1) M_{G}=(1, \ldots, 1) R$ and $(1, \ldots, 1) M_{G_{e}}=(1, \ldots, 1) R L_{\breve{e}}$, we know that

(ii) the set of column sums of $M_{G_{e}}$ is the same as the set of column sums of $M_{G}$.

We also know that if $r$ is a right eigenvector of $M_{G}$ corresponding to an eigenvalue $\lambda$, then $L_{e} R r$ is a right eigenvector of $M_{G_{e}}$ corresponding to $\lambda$, so that

(iii) if $\left(r_{u}\right)_{u \in V_{G}}$ is a right eigenvector of $M_{G}$ corresponding to an eigenvalue $\lambda$, then $\left(s_{v}\right)_{v \in C_{e}}$ with $s_{v}=\sum_{a \in v} r_{t_{G}(a)}$ is that of $M_{G_{e}}$ corresponding to $\lambda$.

If $G$ is irreducible and the spectral radius of $G$ is an integer, say $J$, then $M_{G}$ has the smallest positive integer-valued right eigenvector corresponding to $J$, which Marcus called the smallest right eigenvector corresponding to $J$.

Noting (iii), we see that Marcus's proof in $[\mathrm{M}]$ proves:

Proposition 3.4 (Marcus, $[\mathrm{M}]$ ). Let $G$ be an irreducible graph whose spectral radius is an integer $J$. Assume that the smallest right eigenvector $\left(r_{u}\right)_{u \in V_{G}}$ of $M_{G}$ corresponding to $J$ has a component $r_{u}>1$. Then there exists an equivalence relation $\stackrel{e}{\sim}$ on $A_{G}$ such that

(1) for $a_{1}, a_{2} \in A_{G}, a_{1} \stackrel{e}{\sim} a_{2}$ implies $i_{G}\left(a_{1}\right)=i_{G}\left(a_{1}\right)$, and hence (i) and (ii) above hold for $\lambda=J$;

(2) $M_{G_{e}}$ has the smallest right eigenvector $\left(s_{v}\right)_{v \in C_{e}}$ corresponding to $J$ such that
(a) $\sum_{v \in C_{e}} s_{v}=\sum_{u \in V_{G}} r_{u}$,
(b) $s_{v} \leq \max _{u \in V_{G}} r_{u}$ for all $v \in C_{e}$, and
(c) $\sharp\left\{v \in C_{e} \mid s_{v}=\max _{u \in V_{G}} r_{u}\right\}<\sharp\left\{u \in V_{G} \mid r_{u}=\max _{u \in V_{G}} r_{u}\right\}$.

Marcus's proof of his theorem is completed in the following way.

Let $G$ be an irreducible graph whose spectral radius is an integer $J$. Then using the "transpose" version of the above proposition a necessary number of times, we obtain an irreducible graph $G^{\prime}$ such that $\left(X_{G^{\prime}}, \sigma_{G^{\prime}}\right)$ and $\left(X_{G}, \sigma_{G}\right)$ are topologically conjugate and $M_{G^{\prime}}$ has the left eigenvector corresponding to $J$ with all components equal to 1 (i.e., each column sum of $M_{G^{\prime}}$ is $J$ ). Then using the proposition above a necessary number of times, we obtain from $G^{\prime}$ a graph $G^{\prime \prime}$ such that $\left(X_{G^{\prime \prime}}, \sigma_{G^{\prime \prime}}\right)$ and $\left(X_{G}, \sigma_{G}\right)$ are topologically conjugate and $M_{G^{\prime \prime}}$ has the left and right eigenvectors corresponding to $J$ with all components equal to 1 .

Textile systems $T$ and $T^{\prime}$ are said to be topologically conjugate if there exist topological conjugacies $\psi:\left(X_{T}, \sigma_{T}\right) \rightarrow\left(X_{T^{\prime}}, \sigma_{T^{\prime}}\right)$ and $\Psi:\left(Z_{T}, \varsigma_{T}\right) \rightarrow\left(Z_{T^{\prime}}, \varsigma_{T^{\prime}}\right)$ such that $\psi \xi_{T}=\xi_{T^{\prime}} \Psi$ and $\psi \eta_{T}=\eta_{T^{\prime}} \Psi$.

For $k \geq 1$, a left (resp. right) resolving graph-homomorphism $h: \Gamma \rightarrow G$ is said to be $k$-definite if for any path $\bar{w} \in L_{k}(\Gamma), h(\bar{w})$ uniquely determines $i_{\Gamma}(\bar{w})$ (resp. $\left.t_{\Gamma}(\bar{w})\right)$. As is easily seen, a left or right resolving graph-homomorphism $h: \Gamma \rightarrow G$ is $k$-definite for some $k \geq 1$ if and only if the 1-block map $\phi_{h}:\left(X_{\Gamma}, \sigma_{\Gamma}\right) \rightarrow\left(X_{G}, \sigma_{G}\right)$ is a conjugacy.

Lemma 3.5. Let $T=(p, q: \Gamma \rightarrow G)$ be a nondegenerate, $q$-biresolving textile system such that $p^{*}$ is 1-definite, where $T^{*}=\left(p^{*}, q^{*}: \Gamma^{*} \rightarrow G^{*}\right)$. Let $\stackrel{e}{\sim}$ be an equivalence relation on $A_{G}$ such that for $a_{1}, a_{2} \in A_{G}$, if $a_{1} \stackrel{e}{\sim} a_{2}$, then $i_{G}\left(a_{1}\right)=$ 
$i_{G}\left(a_{2}\right)$. Then $\left(G_{e}\right.$ and $\psi_{e}$ being as above $)$ there exists a nondegenerate q-biresolving textile system $T_{e}=\left(p_{e}, q_{e}: \Gamma_{e} \rightarrow G_{e}\right)$ satisfying the following:

(1) $T$ and $T_{e}$ are topologically conjugate, more precisely, there exists a conjugacy (a bipartite code) $\Psi_{e}: Z_{T} \rightarrow Z_{T_{e}}$ such that $\psi_{e} \xi_{T}=\xi_{T_{e}} \Psi_{e}$ and $\psi_{e} \eta_{T}=\eta_{T_{e}} \Psi_{e}$.

(2) $T_{e}^{*}$ is onesided $1-1$ and if $T^{*}$ is $1-1$, then so is $T_{e}^{*}$.

Proof. We use the same method as in [N2, the proof of Lemma 7.14].

Let $\stackrel{E}{\sim}$ be the equivalence relation on $A_{\Gamma}$ defined as follows. For $\alpha_{1}, \alpha_{2} \in A_{\Gamma}$,

$$
\alpha_{1} \stackrel{E}{\sim} \alpha_{2} \stackrel{\text { def }}{\Leftrightarrow}\left(i_{\Gamma}\left(\alpha_{1}\right)=i_{\Gamma}\left(\alpha_{2}\right)\right) \wedge\left(q_{A}\left(\alpha_{1}\right) \stackrel{e}{\sim} q_{A}\left(\alpha_{2}\right)\right) .
$$

Let $E$ be the canonical map of $\stackrel{E}{\sim}$ and let $C_{E}$ be the set of equivalence classes $E(\alpha), \alpha \in A_{\Gamma}$. Let $\bar{C}_{E}=\left\{\bar{E}(\alpha) \mid \alpha \in A_{\Gamma}\right\}$, where $\bar{E}(\alpha)$ denotes a copy of $E(\alpha)$. Let $\Delta$ be the bipartite graph defined for $\stackrel{E}{\sim}$ in the same way that $H_{e}$ was defined for $\stackrel{e}{\sim}$. Actually, let $A_{\Delta}=A_{\Gamma} \cup \bar{C}_{E}$, let $V_{\Delta}=V_{\Gamma} \cup C_{E}$ and define $i_{\Delta}$ and $t_{\Delta}$ by

$$
i_{\Gamma}(\alpha) \stackrel{i_{\Delta}}{\longleftrightarrow} \bar{E}(\alpha) \stackrel{t_{\Delta}}{\longmapsto} E(\alpha), \quad E(\alpha) \stackrel{i_{\Delta}}{\longleftrightarrow} \alpha \stackrel{t_{\Delta}}{\longmapsto} t_{\Gamma}(\alpha), \quad \alpha \in A_{\Gamma} .
$$

We define a "bipartite" textile system (see $\underline{\mathrm{As}}$ ) $T_{e}^{b}=\left(p_{e}^{b}, q_{e}^{b}: \Delta \rightarrow H_{e}\right)$ by

$$
\begin{aligned}
\left(p_{e}^{b}\right)_{A}(\bar{E}(\alpha)) & =\bar{e}\left(p_{A}(\alpha)\right), & & \left(p_{e}^{b}\right)_{A}(\alpha)=p_{A}(\alpha), \\
\left(q_{e}^{b}\right)_{A}(\bar{E}(\alpha)) & =\bar{e}\left(q_{A}(\alpha)\right), & \left(q_{e}^{b}\right)_{A}(\alpha) & =q_{A}(\alpha)
\end{aligned}
$$

with $\alpha \in A_{\Gamma}$. These $p_{e}^{b}$ and $q_{e}^{b}$ are well defined. For if $\alpha_{1} \stackrel{E}{\sim} \alpha_{2}$ with $\alpha_{1}, \alpha_{2} \in A_{\Gamma}$, then $i_{\Gamma}\left(\alpha_{1}\right)=i_{\Gamma}\left(\alpha_{2}\right)$ and $q_{A}\left(\alpha_{1}\right) \stackrel{e}{\sim} q_{A}\left(\alpha_{2}\right)$, the former of which implies $p_{A}\left(\alpha_{1}\right)=$ $p_{A}\left(\alpha_{2}\right)$ because $p^{*}$ is a 1-definite left resolving graph homomorphism.

Consider the second product-power $\left(T_{e}^{b}\right)^{(2)}$. This is considered to be the disjoint union of the textile systems $\hat{T}_{e}^{b}=\left(\dot{p}_{e}^{b}, \hat{q}_{e}^{b}: \dot{\Delta} \rightarrow \dot{H}_{e}\right)$ and $\grave{T}_{e}^{b}=\left(\grave{p}_{e}^{b}, \grave{q}_{e}^{b}: \grave{\Delta} \rightarrow \grave{H}_{e}\right)$, where $\Delta$ is the graph whose arcs are the paths of length 2 in $\Delta$ going from a vertex in $V_{\Gamma}$ to a vertex in $V_{\Gamma}$ and $\grave{\Delta}$ is the graph whose arcs are the paths of length 2 in $\Delta$ going from a vertex in $C_{E}$ to a vertex in $C_{E}$. Hence $\Delta^{2}=\Delta \sqcup \grave{\Delta}$. Each arc in $\dot{\Delta}$ is of the form $\bar{E}(\alpha) \alpha, \alpha \in A_{\Gamma}$, for which we have

$\left(\dot{p}_{e}^{b}\right)_{A}(\bar{E}(\alpha) \alpha)=\bar{e}\left(p_{A}(\alpha)\right) p_{A}(\alpha) \in A_{\hat{H}_{e}}$ and $\left(\dot{q}_{e}^{b}\right)_{A}(\bar{E}(\alpha) \alpha)=\bar{e}\left(q_{A}(\alpha)\right) q_{A}(\alpha) \in A_{\dot{H}_{e}}$.

Each arc in $\grave{\Delta}$ is of the form $\alpha \bar{E}(\beta), \alpha \beta \in L_{2}(\Gamma), \alpha, \beta \in A_{\Gamma}$, for which we have

$\left(\grave{p}_{e}^{b}\right)_{A}(\alpha \bar{E}(\beta))=p_{A}(\alpha) \bar{e}\left(p_{A}(\beta)\right) \in A_{\grave{H}_{e}}$ and $\left(\grave{q}_{e}^{b}\right)_{A}(\alpha \bar{E}(\beta))=q_{A}(\alpha) \bar{e}\left(q_{A}(\beta)\right) \in A_{\grave{H}_{e}}$.

There exists a graph-isomorphism $k_{E}: \Gamma \rightarrow \Delta$ given by $\left(k_{E}\right)_{A}(\alpha)=\bar{E}(\alpha) \alpha, \alpha \in$ $A_{\Gamma} . T$ and $T_{e}^{b}$ are "isomorphic" through the "textile-system-isomorphism" $\left(k_{E}, k_{e}\right)$, because $p_{e}^{b} k_{E}=k_{e} p$ and $\dot{q}_{e}^{b} k_{E}=k_{e} q$ (see [As]).

If we define a "bipartite" conjugacy $\zeta_{E}:\left(X_{\dot{\Delta}}, \sigma_{\dot{\Delta}}\right) \rightarrow\left(X_{\grave{\Delta}}, \sigma_{\grave{\Delta}}\right)$ by

$$
\left(\bar{E}\left(\alpha_{j}\right) \alpha_{j}\right)_{j \in \mathbf{Z}} \stackrel{\zeta_{E}}{\longmapsto}\left(\alpha_{j} \bar{E}\left(\alpha_{j+1}\right)\right)_{j \in \mathbf{Z}}, \quad\left(\alpha_{j}\right)_{j \in \mathbf{Z}} \in X_{\Gamma},
$$


then we have the commutative diagram

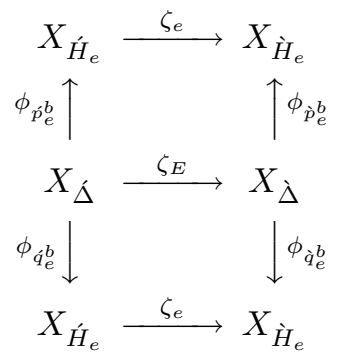

Therefore if we define $T_{e}=\grave{T}_{e}^{b}$ and $\Psi_{e}=\zeta_{E} \kappa_{E}$ with $\kappa_{E}=\phi_{k_{E}}$, then we have $\psi_{e} \xi_{T}=\xi_{T_{e}} \Psi_{e}$ and $\psi_{e} \eta_{T}=\eta_{T_{e}} \Psi_{e}$, and hence $T_{e}$ satisfies (1).

To see that $T_{e}=\grave{T}_{e}^{b}$ is nondegenerate, it suffices to note that $T_{e}^{b}$ is nondegenerate, which follows from the fact that $T_{e}^{b}$ is nondegenerate.

Next we prove that $T_{e}$ is $q$-biresolving. To do this, it suffices to show that $T_{e}^{b}$ is $q$-biresolving. Let $\bar{u} \in V_{\Delta}$. Then $\bar{u} \in V_{\Gamma}$ or $\bar{u} \in C_{E}$. First consider the case that $\bar{u} \in V_{\Gamma}$. Then $\left(q_{e}^{b}\right)_{V}(\bar{u})=q_{V}(\bar{u})$. Each arc in $H_{e}$ starting from $q_{V}(\bar{u})$ is of the form $\bar{e}(a)$ with $a \in A_{G}$ and $i_{G}(a)=q_{V}(\bar{u})$. Since $q$ is right resolving, there exists a unique arc $\alpha \in A_{\Gamma}$ such that $i_{\Gamma}(\alpha)=\bar{u}$ and $q_{A}(\alpha)=a$. It follows that $\bar{E}(\alpha)$ is a unique arc in $\Delta$ such that $i_{\Delta}(\bar{E}(\alpha))=\bar{u}$ and $\left(q_{e}^{b}\right)_{A}(\bar{E}(\alpha))=\bar{e}(a)$. Each arc in $H_{e}$ ending in $q_{V}(\bar{u})$ is of the form $b \in A_{G}$ with $t_{G}(b)=q_{V}(\bar{u})$. Since $q$ is left resolving, there exists a unique arc $\beta \in A_{\Gamma}$ such that $t_{\Gamma}(\beta)=\bar{u}$ and $q_{A}(\beta)=b$. This $\beta$ is a unique arc in $A_{\Delta}$ such that $t_{\Delta}(\beta)=\bar{u}$ and $\left(q_{e}^{b}\right)_{A}(\beta)=b$. Next consider the case that $\bar{u} \in C_{E}$. Then $\bar{u}=E(\alpha)$ for some $\alpha \in A_{\Gamma}$, and $\left(q_{e}^{b}\right)_{V}(E(\alpha))=e\left(q_{A}(\alpha)\right)$. In $H_{e}$ only the arc $\bar{e}\left(q_{A}(\alpha)\right)$ ends in the vertex $e\left(q_{A}(\alpha)\right)$, in $\Delta$ only the arc $\bar{E}(\alpha)$ ends in the vertex $E(\alpha)$ and $\left(q_{e}^{b}\right)_{A}(\bar{E}(\alpha))=\bar{e}\left(q_{A}(\alpha)\right)$. Each arc in $H_{e}$ starting from the vertex $e\left(q_{A}(\alpha)\right)$ is of the form $a \in A_{G}$ with $a \stackrel{e}{\sim} q_{A}(\alpha)$. In $G$, a starts from $i_{G}\left(q_{A}(\alpha)\right)=q_{V}\left(i_{\Gamma}(\alpha)\right)$. Since $q$ is right resolving, there exists a unique arc $\gamma$ in $\Gamma$ satisfying $i_{\Gamma}(\gamma)=i_{\Gamma}(\alpha)$ and $q_{A}(\gamma)=a$, which are equivalent to $\gamma \stackrel{E}{\sim} \alpha$ and $q_{A}(\gamma)=a$. Hence, in $\Delta, \gamma$ is a unique arc starting from $E(\alpha)$ with $\left(q_{e}^{b}\right)_{A}(\gamma)=a$. Thus we have seen that $q_{e}^{b}$ is biresolving.

Since $\left(T_{e}^{b}\right)^{*}$ is onesided 1-1 (because $T^{*}$ is onesided 1-1), it follows that $\left(T_{e}^{b}\right)^{*}$ is onesided 1-1, so that $\left(\grave{T}_{e}^{b}\right)^{*}=T_{e}^{*}$ is onesided 1-1. Similarly, if $T^{*}$ is $1-1$, then $T_{e}^{*}$ is 1-1. Thus $T_{e}$ satisfies (2).

The following lemma is easily proved.

Lemma 3.6. If $T_{1}$ and $T_{2}$ are topologically conjugate textile systems, then the $n$-th composition-powers $T_{1}^{n}$ and $T_{2}^{n}$ are topologically conjugate for all $n \geq 1$.

Proposition 3.7. Let $T=(p, q: \Gamma \rightarrow G)$ be a nondegenerate, $q$-biresolving textile system such that $G$ is irreducible, $\lambda_{G}=J \in \mathbf{Z}$ and $T^{*}$ is onesided 1-1. Then there exist $n \geq 1$ and a nondegenerate, q-biresolving textile system $\breve{T}$ over a graph $\breve{G}$ such that

(i) $T^{n}$ and $\breve{T}$ are topologically conjugate,

(ii) $M_{\breve{G}}$ has the right eigenvector with all components 1 corresponding to $J$, and

(iii) $\breve{T}^{*}$ is onesided 1-1. 
If in addition $T^{*}$ is 1-1 with $\phi_{p}$ bounded-to-one and $\phi_{q} N$-to-one, then there exist $n \geq 1$ and a nondegenerate, $q$-biresolving textile system $\breve{T}$ over a graph $\breve{G}$ such that

(I) $T^{n}$ and $\breve{T}$ are topologically conjugate,

(II) $M_{\breve{G}}$ has the left and right eigenvectors with all components 1 corresponding to $J$, and

(III) $\breve{T}^{*}$ is a 1-1 textile system over a graph $\breve{G}^{*}$ such that $M_{\breve{G}^{*}}$ has the left and right eigenvectors with all components 1 corresponding to $N^{n}$.

Proof. Using Marcus's algorithm which is based on Proposition 3.4, for some $m \geq 0$ we obtain irreducible graphs $G_{0}, \ldots, G_{m}$ and equivalence relations $\stackrel{e_{i}}{\sim}$ on $A_{G_{i}}, i=$ $0, \ldots, m-1$, such that $G_{0}=G, G_{i}=\left(G_{i-1}\right)_{e_{i-1}}$ for $i=1, \ldots, m$, the smallest right eigenvector of $M_{G_{m}}$ corresponding to $J$ is equal to $(1, \ldots, 1)^{t}$. Let $T_{0}=T$. Since $T_{0}^{*}=\left(p_{0}^{*}, q_{0}^{*}: \Gamma_{0}^{*} \rightarrow G_{0}^{*}\right)$ is a onesided 1-1, LL textile system, $p_{0}^{*}$ is a $k_{0}$-definite left resolving graph-homomorphism for some $k_{0} \geq 1$. Let $T_{0}^{k_{0}}=\left(\bar{p}_{0}, \bar{q}_{0}: \bar{\Gamma}_{0} \rightarrow G_{0}\right)$ be the $k_{0}$-th composition-power of $T_{0}$. Then $T_{0}^{k_{0}}$ is a nondegenerate $q$-biresolving textile system such that $\bar{p}_{0}^{*}$ is 1 -definite, where $\left(T_{0}^{k_{0}}\right)^{*}=\left(\bar{p}_{0}^{*}, \bar{q}_{0}^{*}: \bar{\Gamma}_{0}^{*} \rightarrow \bar{G}_{0}^{*}\right)$. Hence we can apply Lemma 3.5 to $T_{0}^{k_{0}}$ and obtain a nondegenerate $q$-biresolving textile system $T_{1}=\left(T_{0}^{k_{0}}\right)_{e_{0}}$ over $G_{1}=\left(G_{0}\right)_{e_{0}}$. By Lemma 3.5, $T_{1}^{*}$ is onesided 1-1 and $T_{1}$ and $T_{0}^{k_{0}}$ are topologically conjugate. Since $T_{1}^{*}$ is onesided 1-1, we similarly obtain a nondegenerate $q$-biresolving textile system $T_{2}=\left(T_{1}^{k_{1}}\right)_{e_{1}}$ over $G_{2}=\left(G_{1}\right)_{e_{1}}$ for some $k_{1} \geq 1$. By Lemma 3.5, $T_{2}^{*}$ is onesided 1-1 and $T_{2}$ and $T_{1}^{k_{1}}$ are topologically conjugate. Continuing this way, we have nondegenerate $q$ biresolving textile systems $T_{i}$ over $G_{i}, i=0, \ldots, m$, such that for $i=0, \ldots, m, T_{i}^{*}$ is onesided 1-1 and $T_{i}$ and $\left(T_{i-1}\right)^{k_{i-1}}$ are topologically conjugate for some $k_{i-1} \geq 1$. If we let $n=k_{0} \ldots k_{m-1}$, then it follows from Lemma 3.6 that $T_{m}$ is topologically conjugate to $T^{n}$. Therefore, if we let $\breve{T}=T_{m}$ and $\breve{G}=G_{m}$, then the first part of the proposition is proved.

If in addition $T^{*}$ is $1-1$ in the above, then it follows from Lemma 3.5 that $T_{i}^{*}$ is 1-1 for $i=1, \ldots, m$. Using Marcus's algorithm based on the "transpose" version of Proposition 3.4, we obtain irreducible graphs $G_{0}^{\prime}, \ldots, G_{m^{\prime}}^{\prime}$ and equivalence relations $\stackrel{e_{i}^{\prime}}{\sim}$ on $A_{G_{i}^{\prime}}, i=0, \ldots, m^{\prime}-1$, such that $G_{0}^{\prime}=G_{m}, G_{i}^{\prime}=\left(G_{i-1}^{\prime}\right)_{e_{i-1}^{\prime}}$ for $i=1, \ldots, m^{\prime}$, the smallest right eigenvector of $M_{G_{m^{\prime}}^{\prime}}$ corresponding to $J$ is equal to $(1, \ldots, 1)^{t}$ and the smallest left eigenvector corresponding to $J$ is equal to $(1, \ldots, 1)$. Using the "transpose" version of Lemma 3.5, we have nondegenerate $q$-biresolving textile systems $T_{i}^{\prime}$ over $G_{i}^{\prime}, i=0, \ldots, m^{\prime}$, with $T_{0}^{\prime}=T_{m}$ such that for $i=0, \ldots, m^{\prime},\left(T_{i}^{\prime}\right)^{*}$ is 1-1 and $T_{i}^{\prime}$ and $\left(T_{i-1}^{\prime}\right)^{k_{i-1}^{\prime}}$ are topologically conjugate for some $k_{i-1}^{\prime}$. Let $n=$ $k_{0} \ldots k_{m-1} k_{0}^{\prime} \ldots k_{m^{\prime}-1}^{\prime}$. Then it follows from Lemma 3.6 that $T_{m^{\prime}}$ is topologically conjugate to $T^{n}$. Therefore, if we let $\breve{T}=T_{m^{\prime}}$ and $\breve{G}=G_{m^{\prime}}$, then the second part of the proposition is proved by using Proposition 3.1(1).

Here we describe a method given in [N2, Section 3] to compute "multipliers" of Boyle $[\mathrm{B}$ in terms of textile systems.

Let $h: \Gamma \rightarrow G$ be a graph-homomorphism between irreducible graphs with $\lambda_{G}=\lambda_{\Gamma}=\lambda$, and assume that $\phi_{h}$ is onto. For $U \subset V_{\Gamma}$ and $w \in L_{G}$, define

$$
\begin{aligned}
& S_{+}(U, w)=\left\{t_{\Gamma}(z) \mid z \in L(\Gamma), i_{\Gamma}(z) \in U, h(z)=w\right\}, \\
& S_{-}(w, U)=\left\{i_{\Gamma}(z) \mid z \in L(\Gamma), t_{\Gamma}(z) \in U, h(z)=w\right\} .
\end{aligned}
$$


We call $S_{+}(U, w)$ and $S_{-}(w, U)$ a successor of $U$ and a predecessor of $U$, respectively. A right-compatible set (for $h$ ) and a left-compatible set (for $h$ ) are defined to be a nonempty successor of a singleton of $V_{\Gamma}$ and a nonempty predecessor of a singleton of $V_{\Gamma}$, respectively. Then any successor of a maximal right-compatible set is also a maximal right-compatible set, and any predecessor of a maximal leftcompatible set is also a maximal left-compatible set ([N1]).

Let $l=(l(v))_{v \in V_{G}}$ and $r=(r(v))_{v \in V_{G}}$ be left and right eigenvectors of $M_{G}$ corresponding to $\lambda$. Let $\bar{l}=(\bar{l}(u))_{u \in V_{\Gamma}}$ and $\bar{r}=(\bar{r}(u))_{u \in V_{\Gamma}}$ be left and right eigenvectors of $M_{\Gamma}$ corresponding to $\lambda$. By a similar argument (using the extremal argument of Boyle [B] ) to that in the proof of Lemma 3.2, we see that for any maximal left-compatible sets $U, U^{\prime}$ for $h$ and any maximal right-compatible sets $V, V^{\prime}$ for $h$,

$$
\sum_{u \in U} \frac{\bar{l}(u)}{l\left(h_{V}(u)\right)}=\sum_{u \in U^{\prime}} \frac{\bar{l}(u)}{l\left(h_{V}(u)\right)} \quad \text { and } \quad \sum_{u \in V} \frac{\bar{r}(u)}{r\left(h_{V}(u)\right)}=\sum_{u \in V^{\prime}} \frac{\bar{r}(u)}{r\left(h_{V}(u)\right)} .
$$

Thus we define $L(h, \bar{l}, l)$ to be the first value and $R(h, \bar{r}, r)$ to be the second. Corresponding to the theorem of L. R. Welch ( $\underline{\mathrm{H}}$, Theorem 14.9]), by similar arguments to those in [H, Section 14] (cf. Lemma 3.2) we have

$$
L(h, \bar{l}, l) R(h, \bar{r}, r) \operatorname{deg} \phi_{h}=\frac{\bar{l} \bar{r}}{l r}
$$

where $\operatorname{deg} \phi_{h}=\sharp \phi_{h}^{-1}(\{x\})$ for any bilaterally transitive point $x \in X_{G}$.

Let $T=(p, q: \Gamma \rightarrow G)$ be a nondegenerate textile system with $G$ and $\Gamma$ irreducible and $\lambda_{G}=\lambda_{\Gamma}$. We define

$$
l_{T}=\frac{L(p, \bar{l}, l)}{L(q, \bar{l}, l)}, \quad r_{T}=\frac{R(p, \bar{r}, r)}{R(q, \bar{r}, r)}, \quad \text { and } \quad \operatorname{deg} T=\frac{\operatorname{deg} \phi_{q}}{\operatorname{deg} \phi_{p}} .
$$

Then these are independent of the choice of $l, \bar{l}, r$ and $\bar{r}$, and they are invariants of topological conjugacy of textile systems ([N2, Proposition 5.4]). We have $l_{T} r_{T}=$ $\operatorname{deg} T$.

Let $m, n$ be nonnegative integers. Let $\varphi$ be an onto endomorphism of $(m, n)$-type of an irreducible topological Markov shift $\left(X_{G}, \sigma_{G}\right)$, that is, there exists a "local rule" $f: L_{m+n+1}(G) \rightarrow A_{G}$ such that $\varphi\left(\left(a_{j}\right)_{j \in \mathbf{Z}}\right)=\left(f\left(a_{j-m}\right) \ldots f\left(a_{j+n}\right)\right)_{j \in \mathbf{Z}}$ for $\left(a_{j}\right)_{j \in \mathbf{Z}} \in X_{G}, a_{j} \in A_{G}$. We define a textile system $T_{\varphi}=\left(p_{\varphi}, q_{\varphi}: G^{[m+n+1]} \rightarrow G\right)$ such that for $w=a_{1} \ldots a_{m+n+1} \in L_{m+n+1}(G), a_{j} \in A_{G}, p_{\varphi}(w)=a_{m+1}$ and $q_{\varphi}(w)=f(w)$. Then $T_{\varphi}$ is onesided 1-1 and nondegenerate and $\varphi_{T_{\varphi}}=\varphi$. The "multipliers" $l_{\varphi}$ and $r_{\varphi}$ of Boyle B are equal to $l_{T_{\varphi}}$ and $r_{T_{\varphi}}$, respectively. In fact, if we choose $\bar{l}$ and $\bar{r}$ such that $\bar{l}(u)=l\left(i_{G}(u)\right)$ and $\bar{r}(u)=r\left(t_{G}(u)\right)$ for $u \in$ $V_{G^{[m+n+1]}}=L_{m+n}(G)$, then we see that

$$
l_{\varphi}=\frac{\lambda_{G}^{m}}{L\left(q_{\varphi}, \bar{l}, l\right)}=l_{T_{\varphi}} \quad \text { and } \quad r_{\varphi}=\frac{\lambda_{G}^{n}}{R\left(q_{\varphi}, \bar{r}, r\right)}=r_{T_{\varphi}} .
$$

If $T$ is a onesided 1-1, nondegenerate textile system over an irreducible graph $G$, then

$$
l_{\varphi_{T}}=l_{T} \quad \text { and } \quad r_{\varphi_{T}}=r_{T}
$$

because $T$ and $T_{\varphi_{T}}$ are topologically conjugate textile systems. If $\psi:\left(X_{G}, \sigma_{G}\right) \rightarrow$ $\left(X_{G^{\prime}}, \sigma_{G^{\prime}}\right)$ is a topological conjugacy, where $G^{\prime}$ is an irreducible graph, then there exists a onesided 1-1, nondegenerate textile system $T^{\prime}$ over $G^{\prime}$ such that $T$ and 
$T^{\prime}$ are topologically conjugate and $\varphi_{T^{\prime}}=\psi \varphi_{T} \psi^{-1}$. Hence $T_{\psi \varphi_{T} \psi^{-1}}$ and $T$ are topologically conjugate.

Theorem 3.8 (Boyle, $[\mathrm{B}$ ). Let $\varphi$ be an onto endomorphism of a topologically transitive topological Markov shift $(X, \sigma)$. Then

(1) $l_{\varphi} r_{\varphi}=\operatorname{deg} \varphi$.

(2) If $\varphi^{\prime}$ is an onto endomorphism of $(X, \sigma)$, then $l_{\varphi \varphi^{\prime}}=l_{\varphi} l_{\varphi^{\prime}}$ and $r_{\varphi \varphi^{\prime}}=$ $r_{\varphi} r_{\varphi^{\prime}}$.

(3) $l_{\varphi}$ and $r_{\varphi}$ are invariants of topological conjugacy of endomorphisms, that is, for any topological conjugacy $\psi:(X, \sigma) \rightarrow\left(X^{\prime}, \sigma^{\prime}\right)$ onto a topological Markov shift, $l_{\psi \varphi \psi^{-1}}=l_{\varphi}$ and $r_{\psi \varphi \psi^{-1}}=r_{\varphi}$.

Proposition 3.9. Let $T=(p, q: \Gamma \rightarrow G)$ be a onesided 1-1, nondegenerate, $q$ biresolving textile system with $G$ irreducible and aperiodic, $\varphi_{T} N$-to-one and $T^{*}$ onesided 1-1. Then

(1) $l_{\varphi_{T}}$ and $r_{\varphi_{T}}$ are integers, and $N$ and $r_{\varphi_{T}}$ are divisible by the same primes.

(2) If $T^{*}$ is $1-1$, then $l_{\varphi_{T}}$ and $r_{\varphi_{T}}$ are integers such that all $N, l_{\varphi_{T}}$ and $r_{\varphi_{T}}$ are divisible by the same primes, and in particular if a prime $p$ divides $N$, then $p^{2}$ divides $N$.

Proof. We use the same argument as in the proof of Proposition 5.4 of [N4].

Since T is $q$-biresolving, $T^{*}=\left(p^{*}, q^{*}: \Gamma^{*} \rightarrow G^{*}\right)$ is LL. Since $\xi_{T^{*}}$ is $1-1, p^{*}$ is a $k$ definite left resolving graph-homomorphism for some $k \geq 1$. Let $\bar{T}=(\bar{p}, \bar{q}: \bar{\Gamma} \rightarrow G)$ be the $k$-th composition-power $T^{k}$ of $T$. Then $\bar{q}$ is biresolving. Since $p^{*}$ is a $k$ definite left resolving graph-homomorphism, it follows that for any $\operatorname{arcs} \alpha, \alpha^{\prime}$ in $\bar{\Gamma}$, $i_{\bar{\Gamma}}(\alpha)=i_{\bar{\Gamma}}\left(\alpha^{\prime}\right)$ implies $\bar{p}_{A}(\alpha)=\bar{p}_{A}\left(\alpha^{\prime}\right)$. Hence we define $F: V_{\bar{\Gamma}} \rightarrow A_{G}$ by

$$
F\left(i_{\bar{\Gamma}}(\alpha)\right)=\bar{p}_{A}(\alpha), \quad \alpha \in A_{\bar{\Gamma}} .
$$

Let $\overline{\bar{T}}=(\overline{\bar{p}}, \overline{\bar{q}}: \overline{\bar{\Gamma}} \rightarrow G)$ be the second composition-power of $\bar{T}$. Then each arc $\gamma$ in $\overline{\bar{\Gamma}}$ is of the form ${ }_{\beta}^{\alpha}$, where $\alpha$ and $\beta$ are $\operatorname{arcs}$ in $\bar{\Gamma}$ with $\bar{q}_{A}(\alpha)=\bar{p}_{A}(\beta)$. For $\gamma={ }_{\beta}^{\alpha}, \overline{\bar{p}}_{A}(\gamma)=\bar{p}_{A}(\alpha)$ and $\overline{\bar{q}}_{A}(\gamma)=\bar{q}_{A}(\beta)$. Since $\bar{q}$ is biresolving, so is $\overline{\bar{q}}$. Let $\hat{T}=(\hat{p}, \hat{q}: \hat{\Gamma} \rightarrow G)$ be the textile system such that $\hat{\Gamma}$ and $\hat{q}$ are the same as $\overline{\bar{\Gamma}}$ and $\overline{\bar{q}}$, respectively, but $\hat{p}$ is defined as follows. For $\gamma={ }_{\beta}^{\alpha}$ in $A_{\hat{\Gamma}}=A_{\overline{\bar{\Gamma}}}$, let $\hat{p}_{A}(\gamma)=F\left(t_{\bar{\Gamma}}(\alpha)\right)$. Then it is observed that $\overline{\bar{T}}$ weaves a textile $\left(\gamma_{i j}\right)_{i, j \in \mathbf{Z}}$ if and only if $\hat{T}$ weaves a textile $\left(\gamma_{i, j-i}\right)_{i, j \in \mathbf{Z}}$. Thus $\hat{T}$ is a onesided 1-1, nondegenerate, $q$-biresolving textile system over the irreducible, aperiodic graph $G$ such that

$$
\varphi_{\hat{T}}=\varphi_{\bar{T}}^{2} \sigma_{\bar{T}}^{-1}=\varphi_{T}^{2 k} \sigma_{T}^{-1} .
$$

Moreover, the same proof as in [N4, the proof of Proposition 5.4] proves that $\hat{T}^{*}$ is $1-1$.

By Corollary 3.3, $\lambda_{G}$ is an integer, say $J$. Hence $M_{G}$ and $M_{\Gamma}$ have left and right eigenvectors corresponding to $J$ such that all the components are in $\mathbf{Q}$. Therefore, $l_{\varphi_{T}}$ and $r_{\varphi_{T}}$ are in $\mathbf{Q}$.

By Proposition 3.7, there exist $n \geq 1$ and a nondegenerate, $q$-biresolving textile system $\breve{T}=(\breve{p}, \breve{q}: \breve{\Gamma} \rightarrow \breve{G})$ such that $T^{n}$ and $\breve{T}$ are topologically conjugate and $r=(1, \ldots, 1)^{t}$ is a right eigenvector of $M_{\breve{G}}$ corresponding to $J$. Since $T$ is a onesided 
1-1, nondegenerate textile system over an irreducible graph, it follows that $\breve{T}$ is onesided 1-1 and $\breve{G}$ and $\breve{\Gamma}$ are irreducible. Since $\breve{q}$ is biresolving, it follows from Proposition 2.3 of [N1] that the column vector $\bar{r}=\left(r\left(\breve{q}_{V}(u)\right)\right)_{u \in V_{\breve{\Gamma}}}$, the components of which are all 1 , is a right eigenvector of $M_{\breve{\Gamma}}$ corresponding to $J=\lambda_{\breve{\Gamma}}=\lambda_{\breve{G}}$. Hence we have $R(\breve{q}, \bar{r}, r)=1$. Thus $r_{\varphi_{\breve{T}}}=r_{\breve{T}}=R(\breve{p}, \bar{r}, r) / R(\breve{q}, \bar{r}, r)=R(\breve{p}, \bar{r}, r)$. Since $R(\breve{p}, \bar{r}, r)$ is equal to the cardinality of the maximal right-compatible set for $\breve{p}$, we see that $r_{\varphi_{\breve{T}}} \in \mathbf{Z}$. Since $\breve{T}$ and $T^{n}$ are topologically conjugate, using Theorem 3.8 we have $\left(r_{\varphi_{T}}\right)^{n}=r_{\varphi_{T}^{n}}=r_{\varphi_{T^{n}}}=r_{T^{n}}=r_{\breve{T}}=r_{\varphi_{\breve{T}}}$. Therefore, since $r_{\varphi_{T}} \in \mathbf{Q}$, we have $r_{\varphi_{T}} \in \mathbf{Z}$.

Applying this result (for $T$ ) to $\hat{T}$, we also have $r_{\varphi_{\hat{T}}} \in \mathbf{Z}$. Applying Theorem 3.8 to (3.6), we have

$$
r_{\varphi_{\hat{T}}}=r_{\varphi_{T}}^{2 k} r_{\sigma_{T}^{-1}}=r_{\varphi_{T}}^{2 k} J^{-1}
$$

Hence $J$ divides $r_{\varphi_{T}}^{2 k}$. Therefore, it follows from Corollary 3.3(3) that every prime dividing $N$ divides $r_{\varphi_{T}}$.

In this stage, we prove (2) preceding the completion of the the proof of (1). To prove (2), assume that $T^{*}$ is $1-1$. Then by the above, $r_{\varphi_{T}}$ is an integer which is divisible by every prime dividing $N$. Moreover, by the "transpose" version of the arguments in the proof above (using the property that $\eta_{T^{*}}$ is 1-1 instead of the property that $\xi_{T^{*}}$ is 1-1), we know that $l_{\varphi_{T}}$ is an integer which is divisible by every prime dividing $N$. Since $l_{\varphi_{T}} r_{\varphi_{T}}=N$, we obtain the proof of (2).

Now we complete the proof of (1). It remains to show that $l_{\varphi_{T}} \in \mathbf{Z}$ and that every prime dividing $r_{\varphi_{T}}$ divides $N$ with only the assumption that $T^{*}$ is onesided 1-1. Applying (2) to $\hat{T}$, we know that $l_{\varphi_{\hat{T}}} \in \mathbf{Z}$. Therefore, since $l_{\varphi_{\hat{T}}}=l_{\varphi_{T}}^{2 k} J$ by (3.6) and Theorem 3.8, we have $l_{\varphi_{T}}^{2 k} J \in \mathbf{Z}$. Since this holds for all sufficiently large $k$ and since $l_{\varphi_{T}} \in \mathbf{Q}$, it follows that $l_{\varphi_{T}} \in \mathbf{Z}$. Since $l_{\varphi_{T}} r_{\varphi_{T}}=N$, every prime dividing $r_{\varphi_{T}}$ divides $N$.

By the proof above, we have the following. (Cf. [N4, Proposition 5.4].)

Proposition 3.10. If $T$ is a onesided 1-1, nondegenerate, $q$-biresolving textile system with $\sigma_{T}$ topologically mixing and $T^{*}$ onesided $1-1$, then there exist $k \geq 1$ and a onesided 1-1, nondegenerate, q-biresolving textile system $\hat{T}$ such that $\varphi_{\hat{T}}=\varphi_{T}^{k} \sigma_{T}^{-1}$ and this is positively expansive.

\section{ExpANSIVE AUTOMORPHISMS OF ONESIDED FULL SHIFTS}

An endomorphism $\varphi$ of a dynamical system $(X, \tau)$, written by $\varphi:(X, \tau) \circlearrowleft$, and another $\varphi^{\prime}:\left(X^{\prime}, \tau^{\prime}\right) \circlearrowleft$ are said to be topologically conjugate if there exists a topological conjugacy $\psi:(X, \tau) \rightarrow\left(X^{\prime}, \tau^{\prime}\right)$ with $\psi \varphi=\varphi^{\prime} \psi$.

Kurka $\mathrm{Ku}$ proved that a positively expansive endomorphism of a transitive topological Markov shift is topologically conjugate to a onesided topological Markov shift. (His proof can be stated as follows. Such an endomorphism is biclosing, so that it is open (see [N1, Theorems 6.3 and 6.5]). Thus use [P, Theorem 1].) Combining this result with [N2, Theorem 3.9(1)], we have:

Theorem 4.1. Let $\varphi$ be a positively expansive endomorphism of a topologically transitive topological Markov shift $(X, \sigma)$. Then there exists a onesided 1-1, nondegenerate, $q$-biresolving textile system $T$ with $T^{*} 1-1$ such that the endomorphisms $\varphi_{T}:\left(X_{T}, \sigma_{T}\right) \circlearrowleft$ and $\varphi$ are topologically conjugate. 
The following theorem proves the third conjecture of Boyle and Maass.

Theorem 4.2. If $\varphi$ is a positively expansive endomorphism of a mixing topological Markov shift and is $N$-to-one, then $l_{\varphi}$ and $r_{\varphi}$ are integers such that all $N, l_{\varphi}$ and $r_{\varphi}$ are divisible by the same primes, and in particular if a prime $p$ divides $N$, then $p^{2}$ divides $N$.

Proof. By Theorem 4.1, Proposition 3.9 and Theorem 3.8.

As was shown in N4, if $\tilde{\varphi}$ is an expansive automorphism of a onesided full shift $\left(A^{\mathbf{N}}, \tilde{\sigma}_{A}\right)$ over an alphabet $A$, then $\left(A^{\mathbf{N}}, \tilde{\varphi}\right)$ is topologically conjugate to a mixing topological Markov shift. Let $\bar{\chi}^{*}$ be any topological conjugacy of $\left(A^{\mathbf{N}}, \tilde{\varphi}\right)$ onto a mixing topological Markov shift $\left(X_{0}, \sigma_{0}\right)$ and let $\varphi_{0}=\bar{\chi}^{*} \tilde{\sigma}_{A}\left(\bar{\chi}^{*}\right)^{-1}$. Define $l_{\tilde{\varphi}}=l_{\varphi_{0}}$ and $r_{\tilde{\varphi}}=r_{\varphi_{0}}(\overline{\mathrm{BM}})$. Then $\varphi_{0}$ is positively expansive and $N$-to-one, where $N=\sharp A$. By Theorem 3.8, $l_{\varphi_{0}}$ and $r_{\varphi_{0}}$ are independent of choice of $\bar{\chi}^{*}$, so that $l_{\tilde{\varphi}}$ and $r_{\tilde{\varphi}}$ are well defined.

Corollary 4.3. Let $\tilde{\varphi}$ be an expansive automorphism of the onesided full shift $\left(A^{\mathbf{N}}, \tilde{\sigma}_{A}\right)$ over the alphabet $A$ of cardinality $N$. Then $l_{\tilde{\varphi}}$ and $r_{\tilde{\varphi}}$ are integers such that all $N, l_{\tilde{\varphi}}$ and $r_{\tilde{\varphi}}$ are divisible by the same primes, and in particular if a prime $p$ divides $N$, then $p^{2}$ divides $N$.

An endomorphism $\tilde{\varphi}:\left(\tilde{X}_{G^{*}}, \tilde{\sigma}_{G^{*}}\right) \circlearrowleft$ of a onesided topological Markov shift over a graph $G^{*}$ is said to be leftmost permutive if $\tilde{\varphi}=\tilde{\varphi}_{f}$ for some mapping (local rule) $f: L_{k+1}\left(G^{*}\right) \rightarrow A_{G^{*}}$ with $k \geq 0$, which induces a left resolving graphhomomorphism of $\left(G^{*}\right)^{[k+1]}$ onto $G^{*}$, where $\tilde{\varphi}_{f}$ is defined by

$$
\tilde{\varphi}_{f}\left(\left(a_{i}\right)_{i \in \mathbf{N}}\right)=\left(f\left(a_{i} \ldots a_{i+k}\right)\right)_{i \in \mathbf{N}}, \quad\left(a_{i}\right)_{i \in \mathbf{N}} \in \tilde{X}_{G^{*}}, a_{i} \in A_{G^{*}} .
$$

Any automorphism of a onesided topological Markov shift is a leftmost permutive endomorphism of the onesided shift. If $\tilde{\varphi}:(\tilde{X}, \tilde{\sigma}) \circlearrowleft$ is a leftmost-permutive endomorphism of a onesided topological Markov shift and $\tilde{\varphi}^{m}$ is topologically transitive for all $m \geq 1$, then there exists a leftmost-permutive endomorphism $\tilde{\varphi}_{0}:\left(A^{\mathbf{N}}, \tilde{\sigma}_{A}\right) \circlearrowleft$ of the onesided full shift over an alphabet $A$ such that $\tilde{\varphi}$ and $\tilde{\varphi}_{0}$ are topologically conjugate ([N4, Corollary 5.3]). Here a leftmost-permutive endomorphism of the onesided full shift $\left(A^{\mathbf{N}}, \tilde{\sigma}_{A}\right)$ means that of $\left(\tilde{X}_{G_{A}}, \tilde{\sigma}_{G_{A}}\right)$, where $G_{A}$ is the one-vertex graph with $A_{G_{A}}=A$. An endomorphism $\tilde{\varphi}:\left(\tilde{X}_{G^{*}}, \tilde{\sigma}_{G^{*}}\right) \circlearrowleft$ of a onesided topological Markov shift is leftmost permutive if and only if $\tilde{\varphi}$ is an LL endomorphism of the shift, that is, there exists a onesided 1-1, LL textile system $T^{*}$ over $G^{*}$ with $\tilde{\varphi}=\tilde{\varphi}_{T^{*}}([\mathrm{~N} 4$, Remark 5.2]).

Remark 4.4. Leftmost permutive or LL endomorphisms of onesided topological Markov shifts are an invariant for topological conjugacy between endomorphisms of onesided subshifts.

Proof. Let $T^{*}=\left(p^{*}, q^{*}: \Gamma^{*} \rightarrow G^{*}\right)$ be a onesided 1-1, LL textile system and let $\tilde{\psi}$ : $\left(\tilde{X}_{G^{*}}, \tilde{\sigma}_{G^{*}}\right) \rightarrow\left(\tilde{X}_{H^{*}}, \tilde{\sigma}_{H^{*}}\right)$ be a topological conjugacy between onesided subshifts, where $H^{*}$ is a nondegenerate graph. Then there exists $k \geq 1$ and $g: L_{k}\left(G^{*}\right) \rightarrow$ $L_{1}\left(H^{*}\right)$ such that $g$ induces a left resolving graph homomorphism of $\left(G^{*}\right)^{[k]}$ onto $H^{*}$ and for $\left(a_{i}\right)_{\in \mathbf{N}} \in \tilde{X}_{G^{*}}$ with $a_{i} \in A_{G^{*}}, \tilde{\psi}\left(\left(a_{i}\right)_{i \in \mathbf{N}}\right)=\left(g\left(a_{i} \ldots a_{i+k-1}\right)\right)_{i \in \mathbf{N}}$. Let $\left(T^{*}\right)^{[k]}=\left(\left(p^{*}\right)^{[k]},\left(q^{*}\right)^{[k]}:\left(\Gamma^{*}\right)^{[k]} \rightarrow\left(G^{*}\right)^{[k]}\right)$ be the higher block textile system of $T^{*}$ of order $k$. Then the textile system $\hat{T}^{*}=\left(g \cdot\left(p^{*}\right)^{[k]}, g \cdot\left(q^{*}\right)^{[k]}:\left(\Gamma^{*}\right)^{[k]} \rightarrow H^{*}\right)$ is a onesided 1-1, LL textile system with $\tilde{\varphi}_{\hat{T}^{*}}=\tilde{\psi} \tilde{\varphi}_{T^{*}} \tilde{\psi}^{-1}$. 
Theorem 4.5. Let $\tilde{\varphi}:\left(A^{\mathbf{N}}, \tilde{\sigma}_{A}\right) \circlearrowleft$ be an expansive leftmost-permutive endomorphism of the onesided full shift over the alphabet $A$ of cardinality $N$. Then there exists a onesided 1-1, nondegenerate, q-biresolving textile system $T$ over an irreducible and aperiodic graph such that $T^{*}$ is onesided $1-1, \varphi_{T}$ is $N$-to-one and $\tilde{\varphi}_{T^{*}}:\left(\tilde{X}_{T^{*}}, \tilde{\sigma}_{T^{*}}\right) \circlearrowleft$ and $\tilde{\varphi}$ are topologically conjugate.

Proof. Let $f: A^{k+1} \rightarrow A$ be a leftmost-permutive local rule with $\tilde{\varphi}=\tilde{\varphi}_{f}$. Let $T_{0}^{*}=\left(p_{0}^{*}, q_{0}^{*}: \Gamma_{0}^{*} \rightarrow G_{A}\right)$ be the textile system such that $\Gamma_{0}^{*}=G_{A}^{[k+1]}$ and for $w=a_{1} \ldots a_{k+1}, a_{i} \in A, p_{0}^{*}(w)=a_{1}$ and $q_{0}^{*}(w)=f(w)$. Then $T_{0}^{*}$ is onesided 1-1 and LL. Let $T_{0}=\left(p_{0}, q_{0}: \Gamma_{0} \rightarrow G_{B}\right)$ be the dual of $T_{0}^{*}$ with $B=A^{k}$. Then $T_{0}$ is onesided 1-1 and $q$-biresolving, $G_{B}$ is irreducible and aperiodic, and $\left(X_{T_{0}^{*}}, \sigma_{T_{0}^{*}}\right)=$ $\left(A^{\mathbf{Z}}, \sigma_{A}\right)$ is topologically mixing. Therefore, by Proposition 3.5 of [N4], $\left(X_{T_{0}}, \sigma_{T_{0}}\right)$ is topologically conjugate to a mixing topological Markov shift.

By the proof of Corollary 3.6 of [N4], there exist $n \geq 1$ and a onesided 1-1, nondegenerate, $q$-biresolving textile system $T=(p, q: \Gamma \rightarrow G)$ with $G$ irreducible and aperiodic such that $U_{T}=U_{T_{0}^{[n]}}$. Since $\tilde{\xi}_{T_{0}^{*}}$ is one-to-one, there are topological conjugacies $\tilde{\psi}:\left(\tilde{X}_{T^{*}}, \tilde{\sigma}_{T^{*}}\right) \rightarrow\left(\tilde{X}_{T_{0}^{*}}, \tilde{\sigma}_{T_{0}^{*}}\right)$ and $\tilde{\Psi}:\left(\tilde{Z}_{T^{*}}, \tilde{\varsigma}_{T^{*}}\right) \rightarrow\left(\tilde{Z}_{T_{0}^{*}}, \tilde{\varsigma}_{T_{0}^{*}}\right)$ with $\tilde{\psi} \tilde{\xi}_{T_{0}^{*}}=\tilde{\xi}_{T^{*}} \tilde{\Psi}$ and $\tilde{\psi} \tilde{\eta}_{T_{0}^{*}}=\tilde{\eta}_{T^{*}} \tilde{\Psi}$ (cf. [N2, the proof of Lemma 2.8]). Hence $T^{*}$ is onesided 1-1 and the endomorphisms $\tilde{\varphi}=\tilde{\varphi}_{T_{0}^{*}}$ and $\tilde{\varphi}_{T^{*}}$ are topologically conjugate.

Since $q$ is biresolving, $\phi_{q}$ is $N^{\prime}$-to-one for some $N^{\prime} \geq 1$, so that $\varphi_{T}$ is $N^{\prime}$-to-one because $T$ is onesided 1-1 and nondegenerate. Since $q_{V}$ is $N^{\prime}$-to-one, the topological entropy of $\left(X_{T^{*}}, \sigma_{T^{*}}\right)$ is $\log N^{\prime}$. The subshift $\left(X_{T^{*}}, \sigma_{T^{*}}\right)$ is topologically conjugate to the full $N$-shift $\left(X_{T_{0}^{*}}, \sigma_{T_{0}^{*}}\right)=\left(A^{\mathbf{Z}}, \sigma_{A}\right)$, so that $N^{\prime}=N$. Thus $\varphi_{T}$ is $N$-toone.

Let $\tilde{\varphi}:(\tilde{X}, \tilde{\sigma}) \circlearrowleft$ be an onto endomorphism of a onesided subshift. Let $\left(\mathcal{O}_{\tilde{\varphi}}, \Sigma_{\tilde{\varphi}}\right)$ be the inverse limit system of $\tilde{\varphi}$, that is, $\mathcal{O}_{\tilde{\varphi}}$ is the compact metric space given by $\mathcal{O}_{\tilde{\varphi}}=\left\{\left(x_{j}\right)_{j \in \mathbf{Z}} \mid \forall j, x_{j} \in \tilde{X}, \tilde{\varphi}\left(x_{j}\right)=x_{j+1}\right\}$ and $d_{\mathcal{O}_{\tilde{\varphi}}}\left(\left(x_{j}\right)_{j \in \mathbf{Z}},\left(y_{j}\right)_{j \in \mathbf{Z}}\right)=$ $\sup \left\{2^{-|j|} d_{\tilde{X}}\left(x_{j}, y_{j}\right) \mid j \in \mathbf{Z}\right\}$, and $\Sigma_{\tilde{\varphi}}: \mathcal{O}_{\tilde{\varphi}} \rightarrow \mathcal{O}_{\tilde{\varphi}} \operatorname{maps}\left(x_{j}\right)_{j \in \mathbf{Z}}$ to $\left(x_{j+1}\right)_{j \in \mathbf{Z}}$. We consider the onto endomorphism $\Phi_{\tilde{\varphi}}:\left(x_{j}\right)_{j \in \mathbf{Z}} \mapsto\left(\tilde{\sigma}\left(x_{j}\right)\right)_{j \in \mathbf{Z}}$ of $\left(\mathcal{O}_{\tilde{\varphi}}, \Sigma_{\tilde{\varphi}}\right)$. If $\tilde{\varphi}$ is expansive (i.e., there is $\delta>0$ such that for any $\left(x_{j}\right)_{j \in \mathbf{Z}},\left(y_{j}\right)_{j \in \mathbf{Z}} \in \mathcal{O}_{\tilde{\varphi}}$, if $d_{\tilde{X}}\left(x_{j}, y_{j}\right) \leq \delta$ for all $j \in \mathbf{Z}$, then $\left.\left(x_{j}\right)_{j \in \mathbf{Z}}=\left(y_{j}\right)_{j \in \mathbf{Z}}\right)$, then there exists an onto endomorphism $\varphi_{0}:\left(X_{0}, \sigma_{0}\right) \circlearrowleft$ of a subshift which is topologically conjugate to $\Phi_{\tilde{\varphi}}:\left(\mathcal{O}_{\tilde{\varphi}}, \Sigma_{\tilde{\varphi}}\right) \circlearrowleft$. If onto endomorphisms $\tilde{\varphi}:(\tilde{X}, \tilde{\sigma}) \circlearrowleft$ and $\tilde{\varphi}^{\prime}:\left(\tilde{X}^{\prime}, \tilde{\sigma}^{\prime}\right) \circlearrowleft$ of onesided subshifts are topologically conjugate, then so are $\Phi_{\tilde{\varphi}}:\left(\mathcal{O}_{\tilde{\varphi}}, \Sigma_{\tilde{\varphi}}\right) \circlearrowleft$ and $\Phi_{\tilde{\varphi}^{\prime}}:\left(\mathcal{O}_{\tilde{\varphi}^{\prime}}, \Sigma_{\tilde{\varphi}^{\prime}}\right) \circlearrowleft$. If in addition these are respectively topologically conjugate to onto endomorphisms $\varphi_{0}:\left(X_{0}, \sigma_{0}\right) \circlearrowleft$ and $\varphi_{0}^{\prime}:\left(X_{0}^{\prime}, \sigma_{0}^{\prime}\right) \circlearrowleft$ of irreducible topological Markov shifts, then $l_{\varphi_{0}}=l_{\varphi_{0}^{\prime}}$ and $r_{\varphi_{0}}=r_{\varphi_{0}^{\prime}}$, by Theorem 3.8. Thus for an onto en$\operatorname{domorphism} \tilde{\varphi}:(\tilde{X}, \tilde{\sigma}) \circlearrowleft$ of a onesided subshift, if $\Phi_{\tilde{\varphi}}:\left(\mathcal{O}_{\tilde{\varphi}}, \Sigma_{\tilde{\varphi}}\right) \circlearrowleft$ is topologically conjugate to an onto endomorphism $\varphi_{0}:\left(X_{0}, \sigma_{0}\right) \circlearrowleft$ of an irreducible topological Markov shift, then we can define $l_{\tilde{\varphi}}$ and $r_{\tilde{\varphi}}$ by $l_{\tilde{\varphi}}=l_{\varphi_{0}}$ and $r_{\tilde{\varphi}}=r_{\varphi_{0}}$ and they are invariants for topological conjugacy between such onto endomorphisms of onesided subshifts.

Theorem 4.6. Let $\tilde{\varphi}$ be an expansive leftmost-permutive endomorphism of the onesided full shift $\left(A^{\mathbf{N}}, \tilde{\sigma}_{A}\right)$ over the alphabet $A$ of cardinality $N$. Then $l_{\tilde{\varphi}}$ and $r_{\tilde{\varphi}}$ are integers, and $N$ and $r_{\tilde{\varphi}}$ are divisible by the same primes.

Proof. By Theorem 4.5, there exists a onesided 1-1, nondegenerate, $q$-biresolving textile system $T$ over an irreducible and aperiodic graph such that $T^{*}$ is onesided 
$1-1, \varphi_{T}$ is $N$-to-one and $\tilde{\varphi}_{T^{*}}:\left(\tilde{X}_{T^{*}}, \tilde{\sigma}_{T^{*}}\right) \circlearrowleft$ and $\tilde{\varphi}$ are topologically conjugate. Since $l_{\tilde{\varphi}}=l_{\varphi_{T}}$ and $r_{\tilde{\varphi}}=r_{\varphi_{T}}$, the theorem follows from Proposition 3.9.

Let $A=\{0,1\}$ and let $B=\{0,1,2\}$. Let $T_{1}=\left(p_{1}, q_{1}: G_{A}^{[3]} \rightarrow G_{A}\right)$ and $T_{2}=\left(p_{2}, q_{2}: G_{B}^{[2]} \rightarrow G_{B}\right)$ be the textile systems defined by $\left(p_{1}\right)_{A}: a_{1} a_{2} a_{3} \mapsto$ $a_{2},\left(q_{1}\right)_{A}: a_{1} a_{2} a_{3} \mapsto a_{1}+a_{3} \bmod 2$ with $a_{j} \in A$ and $\left(p_{2}\right)_{A}: b_{1} b_{2} \mapsto b_{1},\left(q_{2}\right)_{A}:$ $b_{1} b_{2} \mapsto b_{1}+b_{2} \bmod 3$ with $b_{j} \in B$. Then $T_{1}$ and $T_{2}$ are onesided 1-1, nondegenerate and $q$-biresolving. $T_{1}^{*}$ and $T_{2}^{*}$ are onesided 1-1 and LL. If $\varphi_{0}=\varphi_{T_{1}} \times \varphi_{T_{2}}$ and $\tilde{\varphi}=\tilde{\varphi}_{T_{1}^{*}} \times \tilde{\varphi}_{T_{2}^{*}}$, then $\tilde{\varphi}$ is an expansive LL endomorphism of the onesided full 12 -shift with $l_{\tilde{\varphi}}=l_{\varphi_{0}}=2$ and $r_{\tilde{\varphi}}=r_{\varphi_{0}}=6$.

If $T$ is a onesided 1-1, nondegenerate, $q$-biresolving textile system, then we call $\varphi_{T}:\left(X_{T}, \sigma_{T}\right) \circlearrowleft$ a q-biresolving endomorphism of the topological Markov shift $\left(X_{T}, \sigma_{T}\right)([\mathrm{N} 4)$. We are interested in topologically mixing $q$-biresolving endomorphisms of topological Markov shifts, which are also topologically mixing by [N3, Theorem 1.6]. A positively expansive endomorphism of a mixing topological Markov shift is conjugate to a topologically mixing $q$-biresolving endomorphism. The example above gives an example of a topologically mixing $q$-biresolving endomorphism which is not positively expansive. However note Proposition 3.10. It is seen by using Theorem 4.2 that if $\varphi_{1}:\left(A^{\mathbf{Z}}, \sigma_{A}\right) \circlearrowleft$ and $\varphi_{2}:\left(B^{\mathbf{Z}}, \sigma_{B}\right) \circlearrowleft$ are defined by $\varphi_{1}\left(\left(a_{j}\right)_{j \in \mathbf{Z}}\right)=\left(a_{j-1}+a_{j} \bmod 2\right)_{j \in \mathbf{Z}}$ and $\varphi_{2}\left(\left(b_{j}\right)_{j \in \mathbf{Z}}\right)=\left(b_{j}+b_{j+1} \bmod 3\right)_{j \in \mathbf{Z}}$ with $A=\{0,1\}$ and $B=\{0,1,2\}$, then $\varphi=\varphi_{1} \times \varphi_{2}$ is an example of a topologically mixing $q$-biresolving endomorphism such that $\varphi^{m} \sigma^{n}$ is not positively expansive for all $m \in \mathbf{N}$ and $n \in \mathbf{Z}$, where $\sigma=\sigma_{A} \times \sigma_{B}$.

Since topologically mixing, $q$-biresolving endomorphisms of topological Markov shifts are topologically mixing, constant-to-one endomorphisms of mixing topological Markov shifts, constraints for these (see [B], T1] and [T2]) are imposed on them. There are further constraints for them, in particular on their underlying mixing topological Markov shifts, which have not been sufficiently understood. In fact, suppose that a mixing topological Markov shift $\left(X_{G}, \sigma_{G}\right)$ has an $N$-to-one, topologically mixing, $q$-biresolving endomorphism $\varphi$. Then, by Corollary 3.3(1) we know that $\lambda_{G}$ is a rational integer $J$ such that every prime dividing $N$ divides $J$. The result of Boyle and Maass [BM] stated in the Introduction is equivalent to the following. If $\varphi$ is positively expansive, then $\left(X_{G}, \sigma_{G}\right)$ is shift equivalent to some full $J$-shift such that $J$ and $N$ are divisible by the same primes. The second conjecture of Boyle and Maass says that in this result "shift equivalent" can be replaced by "topologically conjugate". Is the result of Boyle and Maass extended to topological Markov shifts having topologically mixing $q$-biresolving endomorphisms? Furthermore, the following is a corrected version of Question 6.5 of [N4].

Question 4.7. Is every topological Markov shift having a topologically mixing $q$-biresolving endomorphism topologically conjugate to a full shift?

\section{REFERENCES}

[AH] N. Aoki and K. Hiraide, Topological Theory of Dynamical Systems, North-Holland, Amsterdam, 1994. MR 1289410 (95m:58095)

[As] H. Aso, Conjugacy of $\mathbf{Z}^{2}$-subshifts and textile systems, Publ. Res. Inst. Math. Sci. 36 (2000), 1-18. MR1749012 (2001a:37016)

[B] M. Boyle, Constraints on the degree of a sofic homomorphism and the induced multiplication of measures on unstable sets, Israel J. Math. 53 (1986), 52-68. MR0861897(88d:28025) 
[BM] M. Boyle and A. Maass, Expansive invertible onesided cellular automata, J. Math. Soc. Japan 52 (2000), 725-740; Erratum, J. Math. Soc. Japan 56 (2004), 309-310. MR1774627 (2001g:37011) MR2027632 (2004i:37019)

[CP] E. Coven and M. Paul, Endomorphisms of irreducible shifts of finite type, Math. Systems Theory 8 (1974), 167-175. MR0383378 (52:4259)

[H] G. A. Hedlund, Endomorphisms and automorphisms of the shift dynamical system, Math. Systems Theory 3 (1969), 320-375. MR0259881 (41:4510)

[Ki] B. Kitchens, Symbolic Dynamics, One-sided, Two-sided and Countable State Markov Chains, Springer-Verlag, 1998. MR.1484730 (98k:58079)

$[\mathrm{Ku}]$ P. Kůrka, Languages, equicontinuity and attractors in cellular automata, Ergodic Theory Dynam. Systems 17 (1997), 417-433. MR 1444061 (98b:58092)

[LM] D. Lind and B. Marcus, An Introduction to Symbolic Dynamics and Coding, Cambridge University Press, 1995. MR 1369092 (97a:58050)

[M] B. Marcus, Factors and extensions of full shifts, Mh. Math. 88 (1979), 239-247. MR0553733 (81g:28023)

[N1] M. Nasu, Constant-to-one and onto global maps of homomorphisms between strongly connected graphs, Ergodic Theory Dynam. Systems 3 (1983), 387-413. MR0741394 (85m:58162)

[N2] M. Nasu, Textile systems for endomorphisms and automorphisms of the shift, Mem. Amer. Math. Soc. 546 (1995). MR1234883 (95i:54051)

[N3] M. Nasu, Endomorphisms of expansive systems on compact metric spaces and the pseudoorbit tracing property, Trans. Amer. Math. Soc. 352 (2000), 4731-4757. MR 1707200 (2001b:54049)

[N4] M. Nasu, The dynamics of expansive invertible onesided cellular automata, Trans. Amer. Math. Soc. 354 (2002), 4067-4084. MR1926865 (2003m:37015)

[P] W. Parry, Symbolic dynamics and transformations of the unit interval, Trans. Amer. Math. Soc. 122 (1966), 368-378. MR.0197683 (33:5846)

[T1] P. Trow, Degrees of constant-to-one factor maps, Proc. Amer. Math. Soc. 103 (1988), 184188. MR0938666 (89m:28030)

[T2] P. Trow, Degrees of finite-to-one factor maps, Israel J. Math. 71 (1990), 229-238. MR:1088817(91m:58052)

[Wa] P. Walters, An Introduction to Ergodic Theory, Springer-Verlag, 1982. MR0648108 (84e:28017)

19-8 TAKaya-TAKAmigaoka 9-ChŌMe, Higashi-Hiroshima 739-2115, JAPAN

E-mail address: nasu@quartz.ocn.ne.jp 